\title{
Effect of geometric parameters on synthetic jet: A review
}

DOI:

$10.1063 / 1.5142408$

\section{Document Version}

Accepted author manuscript

Link to publication record in Manchester Research Explorer

\section{Citation for published version (APA):}

Hong, M. H., Cheng, S. Y., \& Zhong, S. (2020). Effect of geometric parameters on synthetic jet: A review. Physics of Fluids, 32(3), 031301. https://doi.org/10.1063/1.5142408

\section{Published in:}

Physics of Fluids

\section{Citing this paper}

Please note that where the full-text provided on Manchester Research Explorer is the Author Accepted Manuscript or Proof version this may differ from the final Published version. If citing, it is advised that you check and use the publisher's definitive version.

\section{General rights}

Copyright and moral rights for the publications made accessible in the Research Explorer are retained by the authors and/or other copyright owners and it is a condition of accessing publications that users recognise and abide by the legal requirements associated with these rights.

\section{Takedown policy}

If you believe that this document breaches copyright please refer to the University of Manchester's Takedown Procedures [http://man.ac.uk/04Y6Bo] or contact uml.scholarlycommunications@manchester.ac.uk providing relevant details, so we can investigate your claim.

\section{open 2 access}




\title{
TITLE
}

Effect of Geometric Parameters on Synthetic Jet: A Review

\section{AUTHORS}

Mun Hoh Hong ${ }^{1}$, See Yuan Cheng ${ }^{2, *}$, Shan Zhong ${ }^{3}$

1. Faculty of Mechanical Engineering, Universiti Teknikal Malaysia Melaka, Hang Tuah Jaya, 76100 Durian Tunggal, Melaka, Malaysia.

2. Centre for Advanced Research on Energy, Universiti Teknikal Malaysia Melaka, Hang Tuah Jaya, 76100 Durian Tunggal, Melaka, Malaysia.

3. Mechanical and Aeronautical Engineering Division, School of Mechanical, Aerospace and Civil Engineering, The University of Manchester, Oxford Rd, Manchester, M13 9PL, UK

*Author to whom correspondence should be addressed. Electronic mail: cheng@utem.edu.my

\begin{abstract}
A synthetic jet actuator is a fluidic device that produces a jet flow by the periodic ingestion of fluid into and expulsion of fluid out of a cavity across an orifice. Since such a mechanism transfers linear momentum to the fluid without introducing a net mass into the system over an actuation cycle, the synthesised jet is also termed a zero-net-mass-flux jet. Over the last two decades, synthetic jets have been the subject of intense research. It has been shown that the geometric parameters of a synthetic jet actuator can strongly influence the flow characteristics and performance of synthetic jets. The aim of this paper is to provide a comprehensive review of the influence of the geometric parameters of a synthetic jet actuator on the characteristics
\end{abstract}


and performance of synthetic jets. These parameters include the height and diameter of the cavity and the orifice as well as the shape and edge configuration of the orifice.

\subsection{Introduction}

Synthetic jet actuators (SJAs) are devices that are used to produce zero-net-mass-flux (ZNMF) jets by the ingestion of fluid into and expulsion of fluid out of a cavity across an orifice. The ingestion and expulsion processes can be created by various mechanisms, including piezoelectric [1], electromagnetic [2], acoustic [3], and mechanical drivers [4], [5]. Nevertheless, a piezoelectric driver is the most favourable mechanism for flow control and thermal management applications due to its short response time, light weight and highly efficient performance [6].

During the ingestion stage, the diaphragm moves away from the orifice causing the volume of the cavity to increase, thereby leading to a drop in pressure within it. The relatively lower pressure of the inner cavity will cause the fluid of the ambient medium to flow into the cavity. Meanwhile, during the expulsion stage, the diaphragm expels the fluid inside the cavity through the orifice. This expulsion will induce a boundary layer along the inner wall of the orifice to subsequently detach from the wall at the exit due to the sharp edge configuration of the orifice [7]. Next, the separated shear layer will roll up to form a vortex ring. Due to the periodic oscillation of the diaphragm, a train of vortex rings will be produced, which will propagate away from the orifice in response to the self-induced velocity [6].

Due to the transient characteristics of the synthetic jet (SJ), its ubiquitous flow field can be divided into three distinct flow regions (see Fig. 1). First, the near-field region is the part of the flow field that is located in the vicinity of the orifice exit. In this region, the development 
of flow structure is dictated by the formation and conveyance of the discrete vortex rings under a time-periodic reverse flow condition [8]. The next region is known as the transitional region. Here, the vortex rings interact with the ambient fluid and entrain them into the jet. Finally, in the far-field region located further downstream, the structure of the vortex ring will break down into small eddies and the jet flow becomes turbulent [9]. Nevertheless, the vortical structures induced from the SJA can be beneficial for some applications, including for mixing enhancement [10], flow separation control [11]-[20], thermal management [21]-[24], jet vectoring [25]-[27], aero-optics [28], [29], cavity oscillations [30], and shock wave boundary layer (SWBLI) control [31].

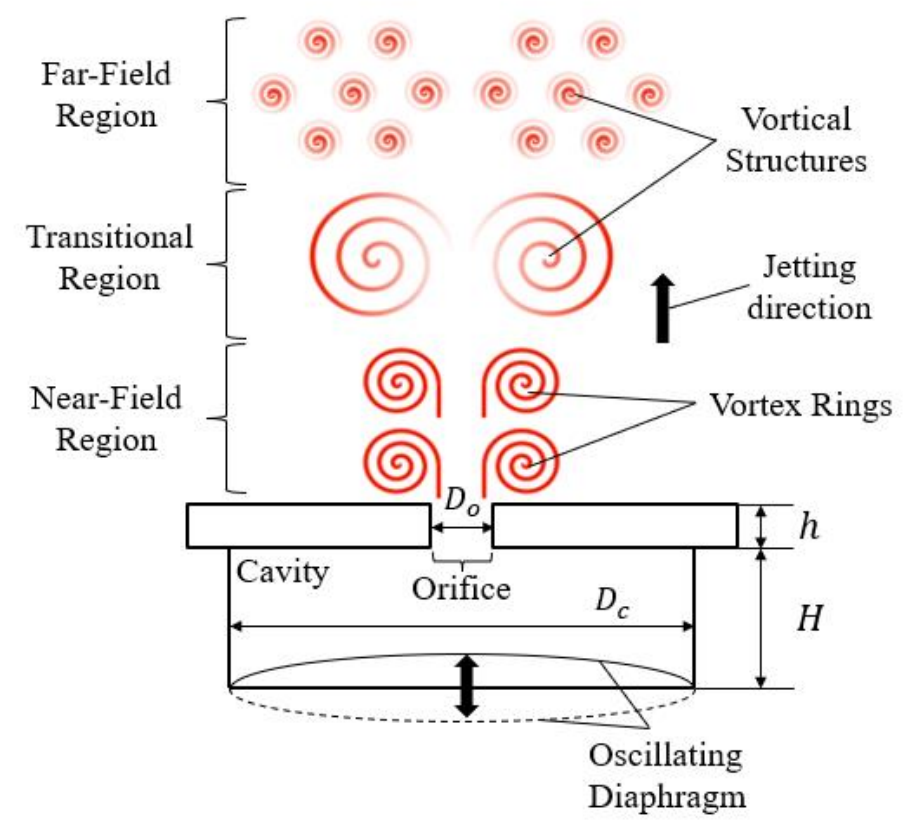

Fig. 1: Labelling of geometrical parameters of synthetic jet 
Table 1: Governing parameters of synthetic jet

\begin{tabular}{ll}
\hline Types of Parameter & Parameter \\
\hline Actuator & Frequency, Amplitude \\
Geometry & Orifice diameter $\left(D_{o}\right)$, Orifice depth $(h)$, \\
& Cavity diameter $\left(D_{c}\right)$, Cavity depth $(H)$, \\
& Orifice shape, etc. (Fig. 1). \\
Fluid & Mean velocity, Density, Kinematic viscosity, \\
& Temperature
\end{tabular}

The physical parameters involved in SJ problems can be divided into three categories, as presented in Table 1. For synthetic jets in quiescent fluids, their behaviour is determined by only two dimensionless parameters, with the most popular choices being the stroke length $\left(\boldsymbol{L}_{\boldsymbol{o}}\right)$ and the Reynolds number (Re), despite variations in how they are defined. According to the slug model adopted by Glezer [32], the stroke length, $\boldsymbol{L}_{\boldsymbol{o}}$ of a synthetic jet is defined as the length of the ejected fluid column from the orifice exit during the expulsion stroke:

$L_{o}=\bar{U}_{e} T$

where, $T=$ time period of the excitation cycle, and $\bar{U}_{e}=$ time-averaged expulsion velocity over one period [33], as shown in Eq. (2):

$\bar{U}_{e}=\frac{1}{T} \int_{0}^{\frac{T}{2}} u_{o}(t) d t$

where, $u_{o}(t)$ refers to the instantaneous stream-wise space averaged velocity at the orifice exit plane. The jet development and vortical evolution are predominantly affected by two nondimensional parameters, i.e., the stroke length and Reynolds number, as defined below:

$L_{\bar{U}_{e}}=\frac{L_{o}}{D_{o}}=\frac{\bar{U}_{e} T}{D_{o}}=\frac{\bar{U}_{e}}{D_{o} f}$ 
$R e_{\bar{U}_{e}}=\frac{\bar{U}_{e} D_{o}}{v}$

where, $D_{o}$ is the orifice diameter, $f$ is the operating frequency of the oscillating diaphragm, and $v$ is the kinematic viscosity of the fluid.

According to Utturkar et al. [34] and Holman et al. [35], the Stokes number (Eq. (5)) and Strouhal number (Eq. (6)) are important parameters for determining the SJ formation criterion shown in Eq. (7).

$S=\sqrt{\frac{2 \pi f D_{o}^{2}}{v}}$

$S t=\frac{2 \pi f D_{o}}{\bar{U}_{e}}$

$\frac{1}{S t}=\frac{R e_{\bar{U}_{e}}}{S^{2}}>K$

where, $K$ is the jet formation constant, which is approximately 1 and 0.16 for two-dimensional and axisymmetric synthetic jets, respectively [35].

Depending on the given application, the performance of the SJA may include the maximum jet velocity, mass flow rate, jet spreading rate, etc. Since the introduction of the SJA system as a possible means of flow control by Wiltse and Glezer in 1994, it has become a subject of intense research. A large volume of studies on SJs, with jet orifices of different aspect ratios, shapes and cavity sizes, have been reported. The behaviour of jets varies significantly under the influence of these geometrical parameters. A few reviews on various topics with regard to SJAs are available. These topics include the fundamental aspects [6], [8], [36], [37], experimental measurement techniques [37], numerical modelling approaches [7], [37], and the application of SJAs for heat transfer enhancement [6], [37], separation flow control [6]-[8], [36], [37], jet mixing [6], as well as propulsion [37]. Despite the important influence of the 
geometric parameters on the performance of the SJA, a compilation of research findings on this particular topic remains to be completed. Therefore, this review focused on how these parameters affect the performance of SJAs for SJs in quiescent flows. The contents of the review could serve as a guideline for choosing the geometry of SJAs so as to optimise the effects for a given application, which could vary from having a maximum jet peak velocity for the delivery of momentum to a jet spreading rate so as to increase mixing within a confined space.

The present review is organized as follows: Section 2 introduces the production of SJs with different aspect ratios. Section 3 reviews the influence of different orifice shapes on the characteristics of SJs. Section 4 reviews the impact of the orifice and cavity dimensions on the performance of the jet. Lastly, Section 5 presents the concluding remarks of this review paper.

\subsection{Effect of Aspect Ratio on Axis-switching Behaviour and Flow Characteristics}

During the initial phase of expulsion, the vortex ring emanating from a slotted orifice has a similar distorted cross section that mimics the approximate shape of the orifice. However, as the ring moves downstream, its cross section expands in the direction of the short axis and shrinks in the direction of the long axis (Fig. 2 (bottom)). This phenomenon is termed 'axis switching' due to the fact that the locations of the short and long axes will switch with each other after a certain downstream distance. Axis switching is regarded as the main mechanism responsible for enhancing the mixing in both continuous jets [38]-[40] and synthetic jets [41], [42]. Nevertheless, several studies have found that a change in the aspect ratio of the slotted orifice can alter the axis-switching behaviour and affect the jet velocity. In this section, the effect of different aspect ratios on the axis-switching behaviour and velocity induced by SJAs are reviewed. 


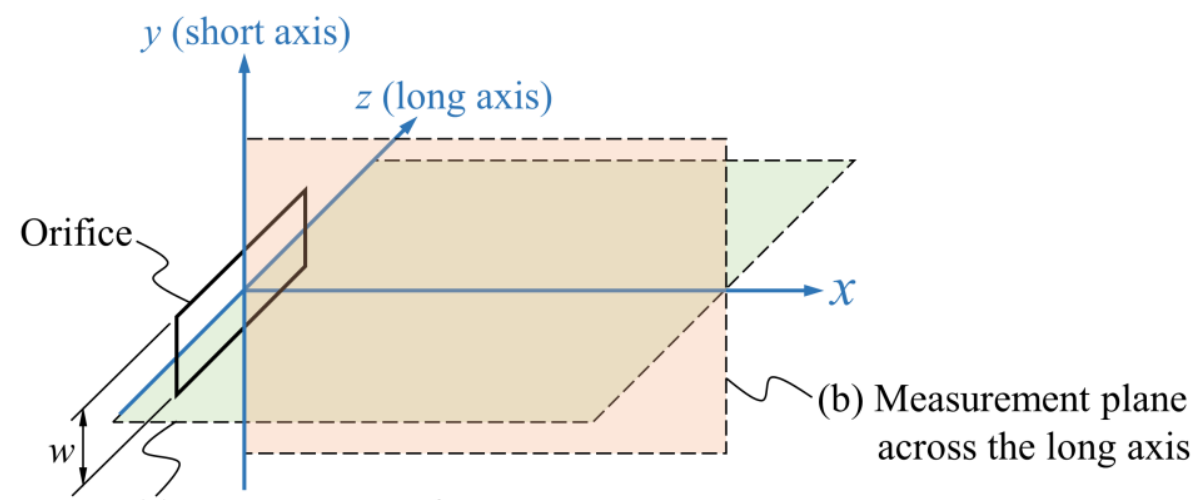

(a) Measurement plane along the long axis

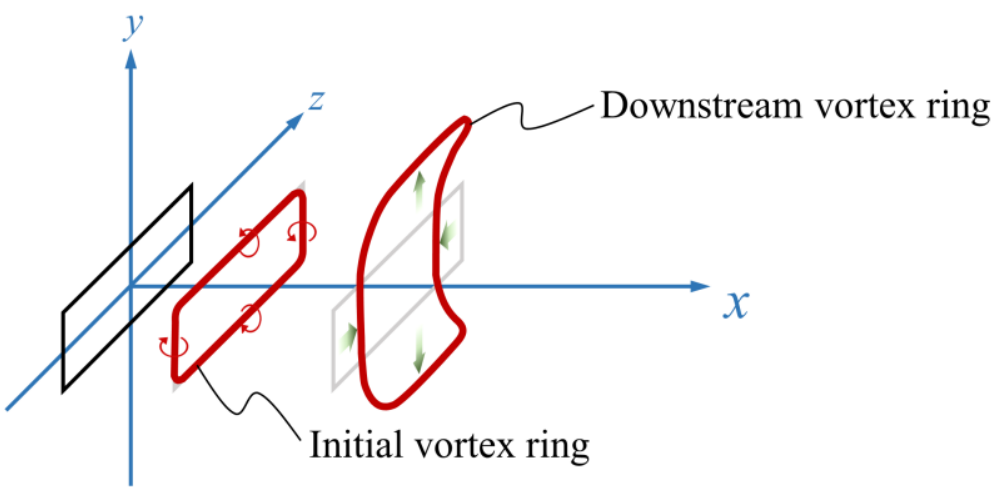

Fig. 2: Experimental measurement plane $(a)$ along and $(b)$ across the long axis of the slotted orifice (Top); Schematic of axis switching (Bottom).

Garcillan et al. [41] conducted an experimental investigation to examine the effect of the aspect ratio of a slotted orifice on the jet flow in a quiescent condition by utilizing the SJA rig of Crook et al. [43]. The jets issuing from orifices with aspect ratios of $\mathrm{AR}=1,5,10$ and 16 were compared, while the cavity geometry and orifice exit area were held as constant. The results showed that as the aspect ratio increased, the axis-switching behaviour became more apparent. In addition, increasing the aspect ratio led to a higher jet exit velocity for a fixed diaphragm displacement. It was explained that for a constant exit area, as the aspect ratio increases, the boundary layer along the orifice duct will occupy a larger portion of the orifice 
duct and cause an increase in the jet exit velocity (i.e. obeying continuity). However, the vortex ring that emanates from an orifice with a larger aspect ratio will undergo a more intense vortex rearrangement process so as to settle into a stable axisymmetric structure, subsequently leading to a higher velocity decay rate.

With the aid of Direct Numerical Simulation (DNS), Ravi et al. [44] examined the flow generated by the SJAs with different slotted orifice aspect ratio. At a constant Reynolds number, i.e. $R e_{J}=227$, the aspect ratio used in the study were $\mathrm{AR}=1,2$ and 4 (note that the Reynolds number was defined as $R e_{J}=\frac{\bar{V}_{j} L_{j}}{v}$, where $\bar{V}_{j}=$ average jet velocity and $L_{j}=$ jet area based length scale, $\left.(w \times l)^{\frac{1}{2}}\right)$. Their results revealed that the axis switching behaviour had grown vigorously at increased aspect ratio, which is in agreement with the experimental results of Garcillan et al. [41]. In addition, they also found that an increased aspect ratio had led to a more parabolic normalized jet exit velocity profile during expulsion and ingestion phases, although with no notable change in the peak value.

Amitay and Cannelle [12] experimentally investigated the effect of large aspect ratio orifice on the streamwise and spanwise flow fields of finite span SJs. Three aspect ratio, i.e. $\mathrm{AR}=50.8,76.2$ and 101.6 were compared while the Reynolds number and Stroke ratio were ranged from $85 \leq R e_{\bar{U}_{e}} \leq 364$ and $16.6 \leq L_{\bar{U}_{e}} \leq 49.4$, respectively (note that the Reynolds number and stroke ratio were defined as $R e_{\bar{U}_{e}}=\frac{\bar{U}_{e} w}{v}$ and $L_{\bar{U}_{e}}=\frac{\bar{U}_{e}}{f w}$, respectively in this study). They discovered that the jet flow fields in the perpendicular (Fig. 2a) and parallel (Fig. 2b) planes of the slotted orifice have responded differently to the effect of aspect ratio. In the former, the jet width has become narrower at increased aspect ratio. They explained that the narrower jet width is caused by the effect of smaller edge in which the flow is induced towards the orifice in the parallel plane. In the latter, however, the jet width has become wider with increasing 
aspect ratio. The dissimilar jet width behaviours in the two planes could be attributed to the delaying of axis switching behaviour as the aspect ratio increases [45]. Based on the observation in the perpendicular plane, the result revealed that the normalized peak streamwise velocity has diminished at increased aspect ratio. This tendency can be explained by the subsequent experimental study of Van Buren et al. [45] who reported that the counter-rotating, edge-induced vortex pair have moved far apart from each other and broken into secondary vortices in the larger aspect ratio orifice case, and led to the reduction in the peak streamwise velocity. Moreover, the result of streamwise evolution of the jet showed that a larger aspect ratio orifice has led to higher centreline velocity decay rate, which is supported by Garcillan et al. [41]. They clarified that this is result of smaller three dimensionality effects associated with the finite length of the slit as the aspect ratio increases. Besides, it was found that the increase in aspect ratio has resulted in higher average velocity over the span, which was based on the normalized streamwise velocity components in the parallel plane.

The study of Kim and Garry [46] who employed a modular construction SJA has investigated the effect of aspect ratio at the range of $\mathrm{AR}=7.14,14.29,21.43,28.57,42.86$ and 78.57. The excitation frequencies for all cases were corresponding to the Helmholtz Resonance frequency. Their result showed that the maximum time-averaged velocity has decreased from $\mathrm{AR}=7.14$ to 14.29 before it attained the maximum magnitude at $\mathrm{AR}=21.43$. It was then continued to decrease gradually from $\mathrm{AR}=28.57$ up to 78.57 . This tendency indicates that there is an optimal aspect ratio in which the maximum time-averaged velocity can be generated as discussed by the subsequent study by Van Buren et al. [45]. Note that the maximum timeaveraged velocity was measured on the parallel plane (as shown in Fig. $2 b$ ) with a distance 1 $\mathrm{mm}$ from the orifice exit. 
Krishnan and Mohseni [47] studied the effect of aspect ratio, $(\mathrm{AR}=45$ and 75) for a range of Reynolds number, $R e_{\bar{U}_{o}}$ and dimensionless stroke ratio, $L_{\bar{U}_{o}}$. For the case with AR $=$ 45, the Reynolds number and stroke ratio were ranged from $137 \leq R e_{\bar{U}_{o}} \leq 311$ and $2.5 \leq$ $L_{\bar{U}_{o}} \leq 5.8$, respectively. Whilst, for $\mathrm{AR}=75$, the Reynolds number and stroke ratio were changed from $141 \leq R e_{\bar{U}_{o}} \leq 254$ and $7.5 \leq L_{\bar{U}_{o}} \leq 13.6$, respectively. Note that the Reynolds number is defined as $R e_{\bar{U}_{o}}=\frac{\sqrt{2} L f w}{v}$ and stroke ratio $L_{\bar{U}_{o}}=\frac{0.25 \pi D_{c}^{2} \Delta}{4 l w^{2}}$ in this study. The operating frequencies that correspond to the membrane resonance frequency were held constant for all the cases. Their result indicated that the centreline velocity decay rate measured from the larger aspect ratio was lower than that with smaller aspect ratio. This tendency disagrees with the results of Garcillan et al. [41] and Amitay and Cannelle [12]. It is notable that the range of operating stroke ratio in Krishnan and Mohseni [47] is much lower than the given stroke ratio in the previous experimental work of Amitay and Cannelle [12]. Thus, the effect of operating stroke ratio might be the source of the discrepancy. Another possibility is there might be an optimal aspect ratio for maximum spreading rate. However, despite the wide range of aspect ratio tested across these three studies, only a limited number of levels were used. Hence, the data might not be sufficient to reveal the optimal aspect ratio.

Van Buren et al. [45] investigated the effect of aspect ratio at three settings (i.e. $A R=6$, 12 and 18) by a stereoscopic particle image velocimetry (SPIV). The study was performed under constant Reynolds number, $R e_{\bar{U}_{e}}=615$ and stroke ratio, $L_{\bar{U}_{e}}=8.696$ (note that the Reynolds number and stroke ratio were defined as $R e_{\bar{U}_{e}}=\frac{\bar{U}_{e} w}{v}$ and $L_{\bar{U}_{e}}=\frac{\bar{U}_{e}}{f w}$, respectively in the study). The results show distinct flow field behaviours in the perpendicular (i.e. the xyplane in Fig. 2) and parallel (i.e. the xz-plane) planes of the slotted orifice (Note that the measurement planes are same as the one presented by Amitay and Cannelle [12]). For this experimental study, $x^{*}$ is referred as non-dimensional values of length, $x^{*}=\frac{x}{w}$, where, $x=$ 
streamwise distance and $w=$ slot width. In the perpendicular plane, the initial jet width was wider at the smaller $\mathrm{AR}$ setting (i.e. $\mathrm{AR}=6$ ). However, at further downstream distance (at $x^{*} \approx 10$ ), the jet width as well as the growth rate had dropped below the values obtained from the larger AR settings (i.e. $A R=12$ and 18). In the parallel plane, the case with $A R=6$ has a narrower jet width in the initial stage. It was then spread wider than the cases with larger aspect ratio at further downstream distance (at $x^{*} \approx 7$ ). The downstream location where the converging flow began to diverge has moved further as the aspect ratio increased from $A R=6$ to 12 , and to 18 . Based on the observations from both of the planes, they deduced that the axis switching behaviour tends to occur in further downstream as the aspect ratio increases.

Furthermore, Van Buren et al. [45] revealed that the highest normalized jet centreline velocity was attained in the case of $A R=12$. They explained that the two counter-rotating, edge-induced vortices exert an influence on each other as they move closer towards each other in the smaller aspect ratio case (i.e. $\mathrm{AR}=6$ ), whereas in the larger aspect ratio case (i.e. $\mathrm{AR}=$ 18) the vortex pair are far apart from each other and break down into secondary vortices. As a consequence, both of the effects lead to a lower maximum centreline velocity. A similar result was shown by the aforementioned experimental study of Kim and Garry [46]. Thus, it can be deduced that there is an optimal aspect ratio ( $A R=12$ in this study) which can prevent the edge induced vortices from either moving too close towards each other or far apart from each other, thus results in highest centreline velocity. They also explained that the existence of the two peaks in the centreline velocity profiles is due to the presence of secondary flow structures in the larger aspect ratio cases, i.e. $\mathrm{AR}=12$ and 18. Besides, their results showed that the centreline velocity at $\mathrm{AR}=6$ decays faster than the cases with $\mathrm{AR}=12$ and 18 , which is contradict to the experimental results obtained by Garcillan et al. [41] and Amitay and Cannelle [12] despite the agreement with Krishnan and Mohseni [47]. As mentioned earlier, the effect of stroke ratio which plays a role in regulating the flow field behaviour of SJAs could be the 
factor causing the discrepancy in the results of velocity decay rate between these studies, because the stroke ratios used by Amitay and Cannelle [12] $\left(16.6 \leq L_{\bar{U}_{e}} \leq 49.4\right)$ are higher than the one used by Krishnan and Mohseni [47] $\left(2.5 \leq L_{\bar{U}_{o}} \leq 13.6\right)$ and Van Buren et al. [45] $\left(L_{\bar{U}_{e}}=8.696\right)$.

Finally, Van Buren et al. [48] also conducted an experimental study to compare the flow behaviour for two aspect ratios (i.e. $A R=18$ and 24) under the condition of high Reynolds number. The two aspect ratios were compared under two Reynolds numbers of, $R e_{\bar{U}_{e}}=1150$ and 3450. For this range of aspect ratios and Reynolds numbers, their results revealed that the influence of the aspect ratio is insignificant as the flow behaviour of the SJs is mainly affected by the Reynolds number.

In summary, non-circular jets are susceptible to axis switching. Hence, they exhibit a higher decay rate than circular jets. Moreover, with an increasing aspect ratio, the axisswitching behaviour becomes more vigorous. Nevertheless, for rectangular jets, the jet exit velocity is proportional to the aspect ratio. In addition, when driven at the Helmholtz resonance frequency, there is an optimal aspect ratio for obtaining the maximum time-averaged velocity.

\subsection{Influence of Different Orifice Shapes on Jet Issuing from SJA}

In the literature, the most widely studied orifice configurations were circular and slotted shapes. However, a few studies also included other orifice designs that are less common such as triangular and square shapes. In this section, the comparative studies between circular and other orifice shapes are reviewed, with particular emphasis on how orifice shapes affect the jet velocity characteristics, such as the peak value, and decaying and spreading rates. 
Garcillan et al. [41] experimentally investigated the effect of three different orifice shapes on the flow field behaviour of SJAs. The Reynolds number and stroke length (both based on the peak axial velocity) ranges from 500 to 800 and 6.4 to 9.6 , respectively. The aspect ratio of the rectangular orifices were $\mathrm{AR}=5,10$ and 16 . By using PIV measurements, circular, square, and rectangular jets were compared while the orifice exit area was held constant for all the cases. Their results showed that the rectangular orifice shows a higher axial velocity decay rate than the circular orifice (Fig. 3). This is the consequence of axis switching behaviour of the jet structures produced by rectangular orifice. Moreover, the square and circular orifices show the existence of secondary vortex trailing behind the primary vortex ring. This is due to the excess circulation that cannot be suppressed by the primary vortices. Intriguingly, the centreline jet velocity of the square jet increases faster downstream than the circular jet albeit both of them starting at the same time phase. This is attributed to the additional momentum induced by the vigorous secondary vortex formed in the square orifice. The strong secondary vortex was generated by high concentration of vorticity around the sharp corners. Subsequently, the secondary vortex coalesces with the primary vortex ring and enhances the momentum of the moving ring. 

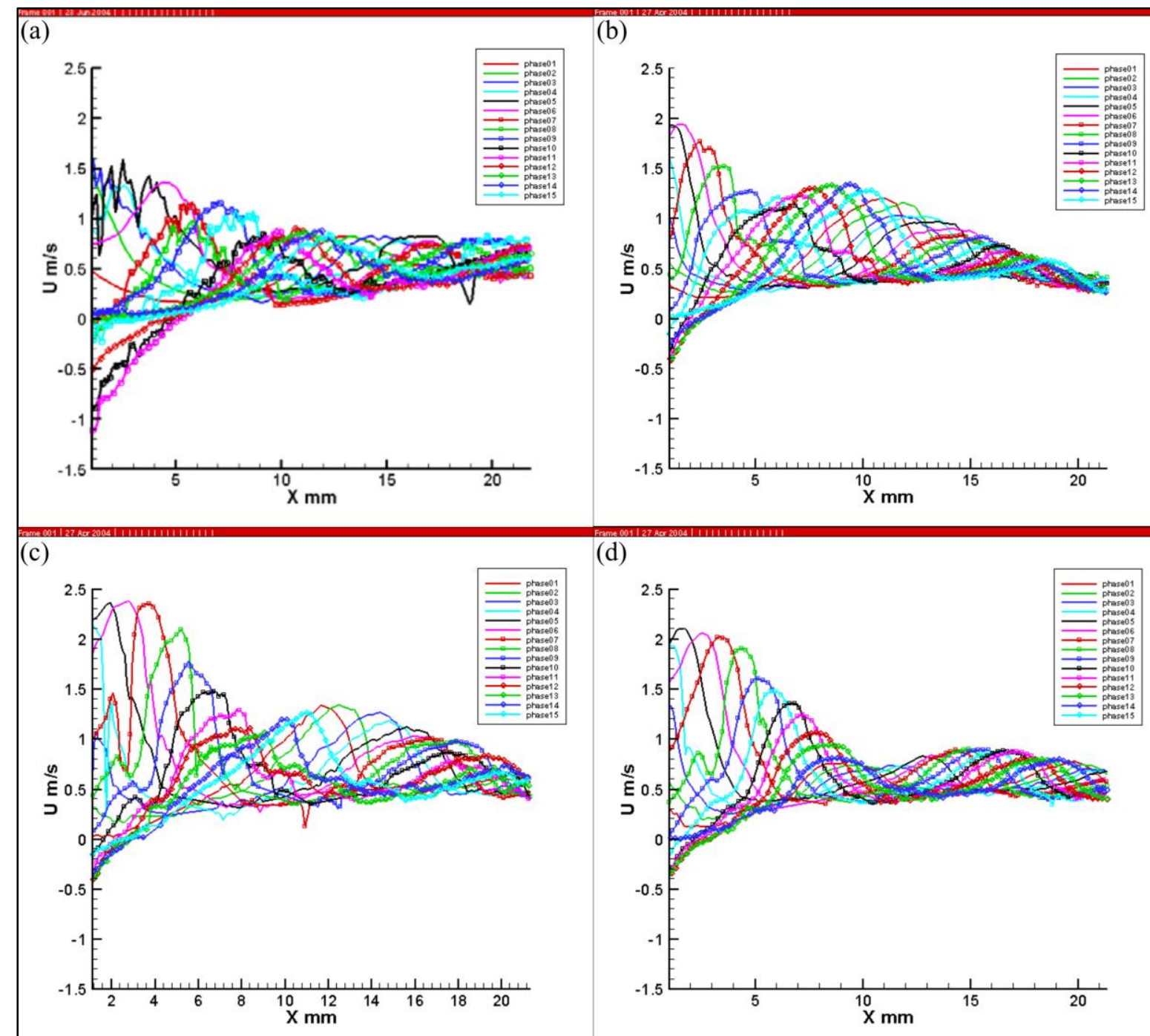

(d)

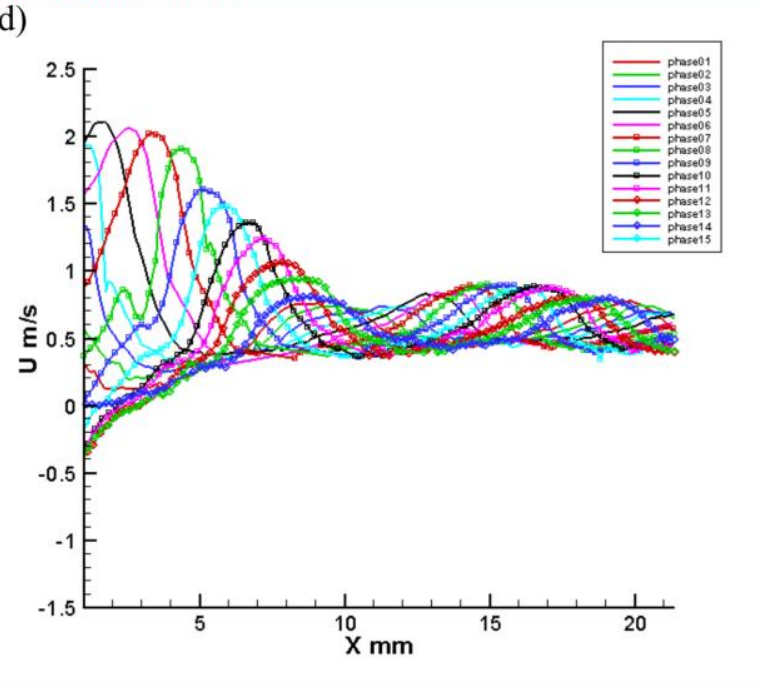

Fig. 3: Velocity variations along the centerline of synthetic jets from the (a) circular orifice and (b) to (c) rectangular orifices; (b) $\mathrm{AR}=5$, (c) $\mathrm{AR}=10$, and (d) $\mathrm{AR}=16$. In all the cases the peak to peak diaphragm displacement is $0.65 \mathrm{~mm}$; Reproduced with permission from $\mathrm{L}$. Garcillan, S. Zhong, Z. Pokusevski, and N. Wood, “A PIV Study of Synthetic Jets with Different Orifice Shape and Orientation,” 2nd AIAA Flow Control Conf., pp. 1-13, 2004. Copyright 2004 University of Manchester.

A similar study was conducted by Oren et al. [42] to identify the suitable orifice shape for thermal control management. For a constant orifice exit area, the velocity characteristics of SJ produced by circular orifice were compared with those by other orifice shapes, namely 
triangular, rectangular and square. In the study, the operating frequencies were coinciding with the Helmholtz Resonance frequency of each of the orifice shape, respectively. Their results indicated that the SJ produced by the circular orifice has a higher peak centreline velocity along the streamwise direction as compared to the one by rectangular orifice. In addition, circular SJ has also exhibited lower centreline velocity decay and spreading rates, which is consistent with the earlier experimental work of Garcillan et al. [41].

Between the jets produced by the triangular and circular orifices, the former has exhibited higher spreading rate along the vertex axis, but similar spreading rate along the flat axis. In addition, the former has also shown higher peak centreline velocity and greater decay rate, which could be associated to the continuation of axis switching behaviour.

By using dye and laser-induced-fluorescene (LIF) flow visualization methods, Wang et al. [49] analysed the influence of orifice aspect ratio on the evolution of SJ. Square (AR =1) and rectangular $(\mathrm{AR}=3$ and 5) orifices were compared with the circular orifice under constant Reynolds number, $R e_{\bar{U}_{e}}=166$ and stroke length, $L_{\bar{U}_{e}}=4.5$. Their results revealed that the vortical area induced from the square orifice was almost similar to that from circular orifice whereas the rectangular orifice has generated the largest vortical area among the three orifice shapes. These outcomes are supported by Oren et al. [42], which indicated that the spreading rates of the jets produced from the circular and square orifices were comparable to each other whereas the spreading rate of the jet produced from the rectangular orifice was higher.

In summary, the spreading rate of a circular jet is comparable to that of a square jet and triangular jet along the flat axis, whereas it is lower than that of a rectangular jet and triangular jet along the vertex axis. In addition, circular jets that do not undergo axis switching will also exhibit a slower velocity decay rate than asymmetric jets. 


\subsection{Impact of Orifice and Cavity Dimensions on Jet Performance}

The dimensions of the orifice and cavity play an important role in the performance of the SJA, which, in principle, is measured by the peak velocity achieved at given diaphragm operating conditions. Typically, the curve of the SJ velocity versus the diaphragm excitation frequency may involve two maxima (Fig. 4(a) and (b)) or a single peak (Fig. 4(c)). The jet velocity reaches the maximum value when the excitation frequency coincides with the two resonance frequencies controlling the actuator, namely, the natural frequency of the piezoelectric diaphragm, and the Helmholtz frequency of the cavity (Gallas et al. [50]). Despite the general trend, Fig. 4 reveals that the peak value varies from case to case, indicating the important effects of the dimensions of the cavity and orifice.

This section first describes the incompressible flow model for estimating the jet velocity. Then, the impact of the orifice depth, cavity height, cavity diameter, and orifice edge configuration on the jet peak velocity, as well as the other performance indicators associated with the jet peak velocity, are reviewed. A discussion on the orifice diameter was omitted for two reasons. First, from a design point of view, the orifice diameter is mainly determined by the application, i.e. the boundary layer thickness or pipe diameter. Normally, it should not be greater than 5\% to minimize flow disturbances. Second, the orifice diameter is used to calculate all the relevant dimensionless parameters. Hence, changing it will result in changes to those parameters and, in most cases, the outcome will only affect the Reynolds number. Thus, a discussion based on the orifice diameter can be misleading. 

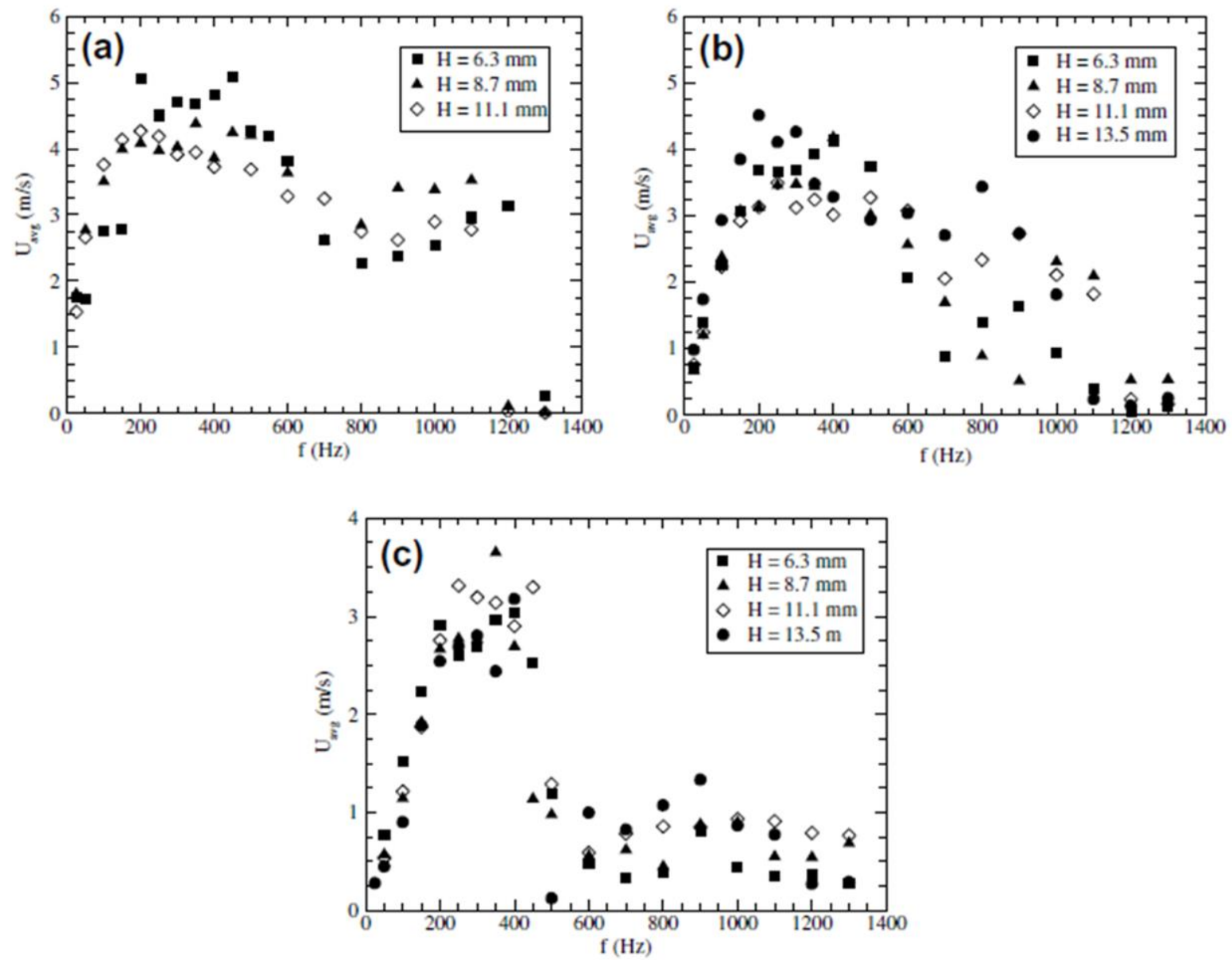

Fig. 4: Variation of average velocity with excitation frequency for different cavity depths and orifice diameters; The orifice diameters are (a) $3 \mathrm{~mm}$, (b) $5 \mathrm{~mm}$, and (c) $8 \mathrm{~mm}$; Reproduced with permission from M. Chaudhari, G. Verma, B. Puranik, and A. Agrawal, "Frequency response of a synthetic jet cavity," Exp. Therm. Fluid Sci., vol. 33, no. 3, pp. 439-448, 2009. Copyright 2008 Elsevier Inc.

\subsection{Estimation of Jet Peak Velocity using an Incompressible Flow Model}

The actuator parameters, such as the frequency and amplitude of an oscillating membrane or piston, play a major role in characterizing the performance of a synthetic jet actuator. According to the incompressible model proposed by Tang and Zhong [51], the space- 
averaged peak velocity can be estimated by using Eq. (8) below, without having to consider modelling the flows inside the cavity and orifice duct.

$u_{\text {peak } \_v}=\frac{\pi}{4} \Delta f\left(\frac{D_{c}}{D_{o}}\right)^{2}$

where, $u_{\text {peak_v }}=$ jet peak velocity, $\Delta=$ peak-to-peak displacement of oscillating diaphragm, $D_{o}=$ orifice diameter, $D_{c}=$ cavity diameter. Based on Eq. (8), the jet peak velocity is only dependent on the oscillating frequency, peak-to-peak displacement of the diaphragm, and ratio of the cavity diameter to the orifice diameter.

The jet peak velocity can increase substantially if the oscillating frequency synchronizes with either the acoustic resonance frequency (Helmholtz resonance frequency) of the cavity or the structural resonance frequency of the diaphragm. However, the structural resonance of the diaphragm was not discussed here since it is determined by its materials and how it is clamped. When the excitation frequency coincides with the Helmholtz resonance frequency, the compressibility effect will become significant and will regulate the exit velocity of the jet [37]. The lumped-element model (LEM) proposed by Gallas et al. [50] enables the relationship between the Helmholtz resonance frequency $\left(f_{H}\right)$ of the cavity and the actuator geometry to be established:

$f_{H}=\left(\frac{1}{2 \pi}\right)\left[\left(\frac{4 \rho h}{3 \pi r_{o}^{2}}+\frac{8 \rho}{3 \pi^{2} r_{o}}\right)\left(\frac{V_{c}}{\rho c^{2}}\right)\right]^{-0.5}$

where, $r_{o}=$ orifice radius, $\rho=$ density of fluid, $h=$ orifice depth, $V_{c}=$ volume of cavity, $c=$ speed of sound. 


\subsection{Impact of Orifice Depth on Jet Velocity}

The orifice depth is one of the important parameters that modulate the flow from the cavity into the ambient medium. In addition, it is directly related to the flow characterization induced by SJAs, as has been presented in numerous studies.

Jabbal et al. [52] investigated the effect of the orifice depth experimentally. The orifice depths were $h=1.25,2.5,5$ and $10 \mathrm{~mm}$ (corresponding to $0.25 D_{o}, 0.5 D_{o}, 1 D_{o}$ and $2 D_{o}$, respectively), while $R e_{\bar{U}_{e}}=559$ and $L_{\bar{U}_{e}}=3.24$. The results revealed that the instantaneous maximum blowing velocity gradually increased from $h / D_{o}=2$ to 1 before it reached a peak at $h / D_{o}=0.5$, followed by a drop at $h / D_{o}=0.25$. It was clarified that a virtual pipe with a smaller diameter and bounded with streamlines was formed inside the orifice duct due to the flow separation at the inlet of the orifice. The location on a virtual pipe where the diameter is the least is termed the vena contracta. At $h / D_{o}=1$ and 2 , the vena contracta remained inside the orifice duct since the flow was able to reattach along the duct wall before reaching the orifice exit plane (Fig. 5a). As the orifice depth decreased, the flow reattachment did not occur and the vena contracta was relocated out of the duct. Therefore, the vortices were ejected in a smaller virtual exit diameter. Subsequently, a higher maximum blowing velocity was shown in the case of $h / D_{o}=0.5$ (Fig. $5 b$ ). However, when the orifice depth was relatively smaller than the size of the orifice, i.e., $h / D_{o}=0.25$, the virtual exit diameter matched the orifice diameter due to the absence of a constriction effect (Fig. 5c). Consequently, it resulted in a lower blowing velocity. 


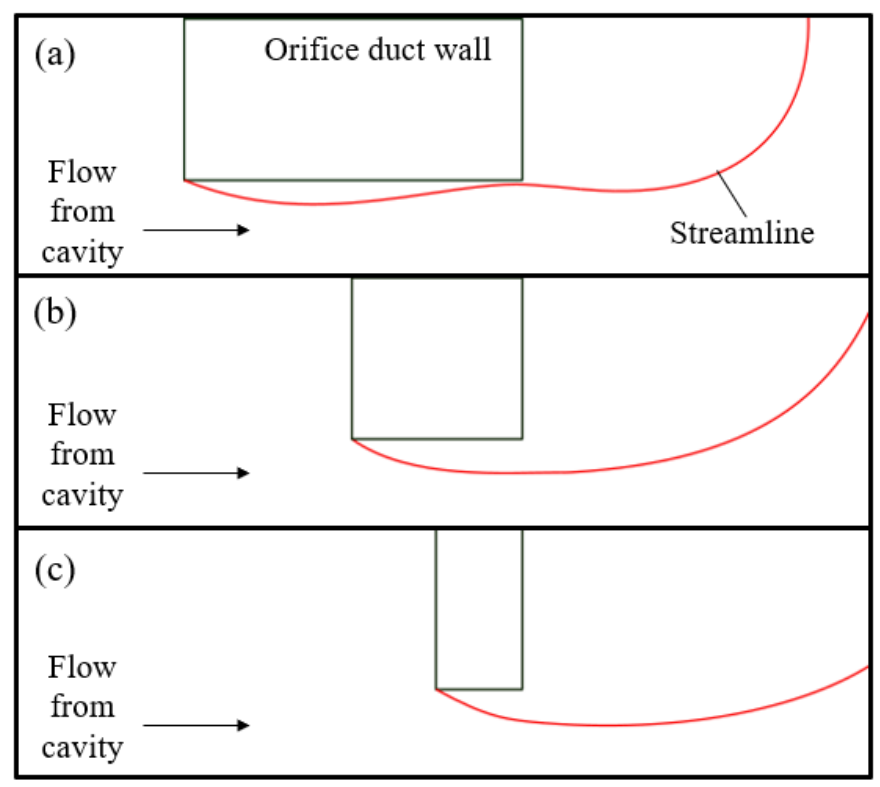

Fig. 5: Flow ejected from orifice exit for $a) h=5 \mathrm{~mm}, b) h=2.5 \mathrm{~mm}$, and $c) h=1.25 \mathrm{~mm}$, which are corresponding to $1 D_{o}, 0.5 D_{o}$, and $0.25 D_{o}$ respectively.

Jain et al. [53] carried up a numerical study to examine the effect of orifice dimensions which include the orifice depth on the SJA flow field. The orifice height of the study were 0.5, 1.5, 2.5 and $3 \mathrm{~mm}$ (corresponding to $0.17 D_{o}, 0.5 D_{o}, 0.83 D_{o}$ and $1 D_{o}$, respectively) while the $R e_{\bar{U}_{e}}$ were between 1453 and 1569 . Their results showed that the maximum jet velocity has risen moderately from $h / D_{o}=0.17$ to 0.5 and remained constant until 0.83 before it decreased at $h / D_{o}=1$. Thus, the observation is in good agreement with the previous experimental results of Jabbal et al. [52] which suggested there is an optimum orifice depth where the maximum velocity can be attained.

The subsequent numerical study by Lv et al. [54] revealed the dependency of timeaveraged blowing velocity on the orifice depth that corresponds to the Helmholtz Resonance frequency. As shown in the numerical results, the larger orifice depth which corresponds to the Helmholtz Resonance frequency will result in a higher maximum time-averaged blowing 
velocity. Based on the relation in Eq. (8), larger orifice depth will correspond to a lower Helmholtz Resonance frequency. At lower Helmholtz Resonance frequency, weak dissipation of gas occurred at the orifice exit. The orifice depth is found to have no significant impact on the blowing velocity. In the case of smaller orifice depth that corresponds to a higher Helmholtz Resonance frequency, improper flow coordination in the cavity and orifice during expulsion and ingestion phases will result in smaller peak time-averaged blowing velocity as compared to the larger orifice depth cases.

Lee and Goldstein [55] employed a Direct Numerical Simulation (DNS) method to investigate the behaviour of 2-D, slotted jets pulsing into a quiescent flow field at different orifice depths. The baseline case defined as a sharp plate of near-zero thickness was compared with $0.4 w, 1.0 w, 2.0 w$ and $2.5 w$ orifice depths (where $w$ is referred to the width of slot) while the $R e_{\bar{U}_{\text {peak }}}=104.2$ and $L_{\bar{U}_{\text {peak }}}=15.924$ were held constant for all the cases (note that $R e_{\bar{U}_{\text {peak }}}$ and $L_{\bar{U}_{\text {peak }}}$ are derived by using slot half-width, $\left.w_{\frac{1}{2}}\right)$. The results indicated that as the orifice depth increased from the baseline case to $2.5 \mathrm{w}$, a higher peak streamwise velocity was reported. This is attributed to the development of thicker boundary layer along the orifice duct wall and enhances the constriction effect along the duct. Hence, the vortices are ejected at a higher exit velocity due to the reduction in the actual slot width at the orifice exit plane.

Van Buren et al. [45] experimentally investigated the effect of orifice depth on the flow field induced by a slotted orifice SJA. The orifice depth, $h$ of the study were 2,4 and $6 \mathrm{~mm}$ while the Reynolds number, $R e_{\bar{U}_{e}}$ and stroke ratio, $L_{\bar{U}_{e}}$ were held constant at 615 and 8.7, respectively (note that the Reynolds number and stroke ratio are defined as $R e_{\bar{U}_{e}}=\frac{\bar{U}_{e} w}{v}$ and $L_{\bar{U}_{e}}=\frac{\bar{U}_{e}}{f w}$, respectively in this study). Their results revealed that at the lowest orifice depth $(h$ $=2 \mathrm{~mm}$ ) setting, the maximum normalized streamwise velocity in the plane across the long 
axis at the near-field region was slightly higher than that obtained at the larger orifice depths. However, at further downstream region, it was surpassed by the values of the larger orifice depth cases ( $h=4$ and $6 \mathrm{~mm}$ ). Meanwhile, the magnitudes of the maximum streamwise velocity of the three orifice depth cases have not exhibited any significant change in the plane along the long axis. This study indicated that the orifice depth has relatively smaller influence on the jet strength in both the near field and far field regions when compared to the effect of aspect ratio under the constant Reynolds number, $R e_{\bar{U}_{e}}$ and stroke ratio, $L_{\bar{U}_{e}}$.

\subsection{Effects of Cavity Dimensions on Jet Velocity}

As a synthetic jet is typically generated by periodically forcing the fluid from the cavity of the SJA through an orifice into the external region and back into the cavity, the geometric parameters of the cavity play an important role in influencing the flow characteristics of the SJ. Two geometric parameters are particularly important, namely, the cavity height and cavity diameter. This section reviews their influence on the jet velocity.

\subsubsection{Influence of Cavity Height on Jet Velocity}

Mallinson et al. [56] investigated the influence of cavity height by experimental and computational approaches. In their study, the cavity diameter and orifice depth were held constant at $36.8 \mathrm{~mm}$ and $1.65 \mathrm{~mm}$, respectively. The Reynolds number and stroke ratio were $73.66 \leq R e_{\bar{U}_{\text {mean }}} \leq 1012.3$ and $2 \leq L_{\bar{U}_{\text {mean }}} \leq 15$, respectively. Whilst, two cavity heights $(H=$ 1.35 and $2.85 \mathrm{~mm})$ and four orifice diameters $\left(D_{o}=0.5,0.75,1.0\right.$, and $\left.2.0 \mathrm{~mm}\right)$ were used. The maximum mean velocity on the jet centre-line at $1 \mathrm{~mm}$ above the orifice as a function of the ratio of orifice diameter to cavity height was examined. The results showed that in general, the 
maximum mean velocity values were lower in the larger cavity height cases. They explained that at larger cavity height, the swept volume by the diaphragm oscillation motion, relative to the cavity volume, becomes smaller. Consequently, lower induced velocity is produced.

By using both the experimental and numerical approaches, Mane et al. [1] studied the effect of cavity height on jet peak velocity. Two different types of piezoelectric composite diaphragm were used, namely, Bimorph and Thunder ${ }^{\circledR}$. The cavity heights of the study were 5.50 and $9.95 \mathrm{~mm}$. Their results showed that at decreased cavity height, the axial jet velocity increased independent of the type of composite diaphragm. As explained by Mane [57], this is because the vortices cease to occur in a shallow cavity and eventually leads to higher mass flow rate and velocity. The findings were supported by Rizzetta et al. [58] who numerically examined the effect of cavity geometry on the flow fields generated by SJA. The cavity heights of the study were 1 and $5 \mathrm{~mm}$ while the constant Reynolds Number, $R e_{\bar{U}_{\text {ref }}}=750$ and stroke ratio, $L_{\bar{U}_{\text {ref }}} \approx 44$ were used. The results showed that, under the same forcing amplitude the case with the shallower cavity height induced a higher time-mean jet exit velocity. In addition, the visualization of the numerical results depicted that a stronger vortex pair was ejected from the orifice when a shallower cavity height was used.

Similar results were obtained from the numerical work of Lee and Goldstein [55]. In their study, the results obtained from the half and quarter cavity height cases were compared to the full cavity height case. All the cases are performed at the constant Reynolds Number, $R e_{\bar{U}_{\text {peak }}}=104.2$ and stroke ratio, $L_{\bar{U}_{\text {peak }}}=15.924$ (note that the $R e_{\bar{U}_{\text {peak }}}$ and $L_{\bar{U}_{\text {peak }}}$ were based on the slot half-width, $\left.w_{\frac{1}{2}}\right)$. They found that as the cavity height decreased, higher velocity of the ejected vortices was produced. They explained that at shallower cavity height, the vorticity which forms in the cavity will move closer to the inner side of the orifice plate, thereby augments the speed of the vertical flow along the inner side of the orifice plate towards 
the orifice exit. As a result of this added momentum, there is a slight narrowing of the effective opening of the orifice. Thus, the vortices will be ejected with relatively higher exit velocity (by continuity of flow).

Jain et al. [53] investigated the influence of different cavity heights $\left(\mathrm{H}=0.5 D_{o}, 0.83 D_{o}\right.$, $1.66 D_{o}, 2.33 D_{o}, 3.17 D_{o}, 4 D_{o}$ and $6 D_{o} ; D_{o}=3 \mathrm{~mm}$ ) on jet flow field numerically. Their results indicated that when the cavity height was varied from $0.5 D_{o}$ to $4 D_{o}$, the peak velocity of the jet dropped by only a small amount. However, the duration of the peak velocity tended to prolong at shallower cavity (Fig. 6). They explained that the extended peak velocity duration is caused by the smaller phase difference between the diaphragm oscillating motion and velocity cycle at the orifice exit. Such circumstance allows the diaphragm and velocity cycle to oscillate in the same direction when the peak velocity occurs, and thus, causing it to last longer. In the contrary, a larger phase difference which occurs in a deeper cavity case will allow the diaphragm to move in direction opposite to the velocity cycle at the time when jet velocity reaches its peak. This circumstance will result in a jet flow with lower peak velocity and shorter duration. 


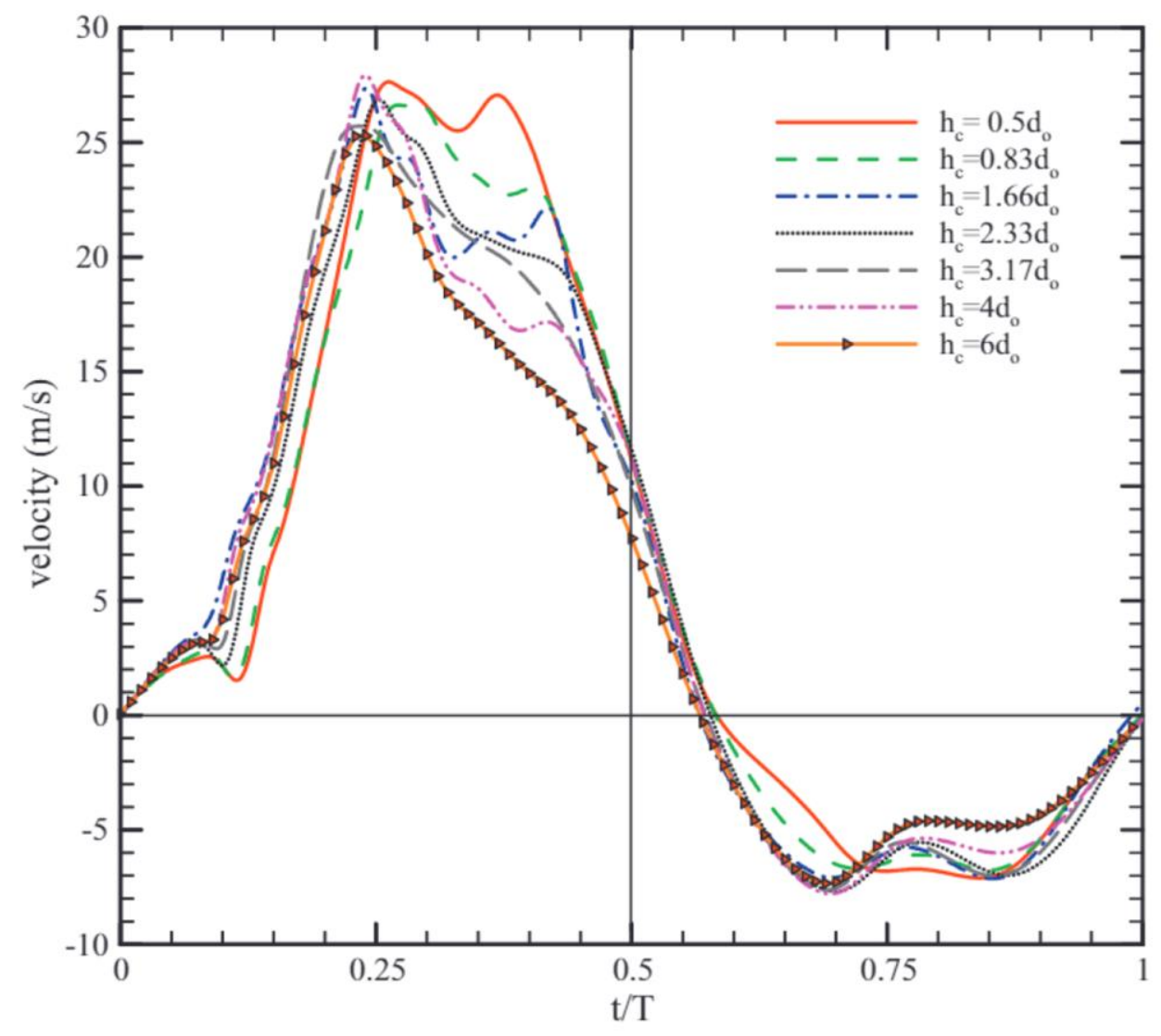

Fig. 6: Effect of cavity height on the velocity at the orifice exit; Reproduced with permission from M. Jain, B. Puranik, and A. Agrawal, “A numerical investigation of effects of cavity and orifice parameters on the characteristics of a synthetic jet flow," Sensors Actuators A. Phys., vol. 165, pp. 351-366, 2011. Copyright 2010 Elsevier B.V.

Jabbal et al. [52] studied experimentally the effect of cavity height on the vortex circulation of circular synthetic jets in a quiescent flow. The cavity heights of their study were 5, 10 and $20 \mathrm{~mm}$ while the Reynolds Number and stroke ratio were held constant at $\operatorname{Re}_{\bar{U}_{e}}=$ 559 and $L_{\bar{U}_{e}}=3.24$, respectively. Surprisingly, the results depicted that as the cavity height decreased, the instantaneous maximum blowing velocity decreased. Although the result trend agreed with the findings of Crook and Wood [43], it was in disagreement with the experimental works of Mallinson et al. [56] and Mane et al. [1], including the numerical studies of Rizzetta 
et al. [58], Lee and Goldstein [55] and Jain et al. [53]. Jabbal et al. [52] attributed the contradiction between their work and the previous studies to the existence of Helmholtz Resonance effect. They explained that when the diaphragm excitation frequency coinciding with the Helmholtz Resonance frequency, the pressure variation augments in the cavity and leads to a higher mass flow through the orifice exit. In their experimental study, the Helmholtz Resonance frequency decreased and approached the operating frequency with larger cavity height. Therefore, resulted in a higher instantaneous maximum blowing velocity at the orifice exit.

There are also several studies investigated the effect of cavity height on the SJ characteristics under the condition where the diaphragm excitation frequency is coinciding with the Helmholtz Resonance frequency of the cavity. In this regards, Chaudhari et al. [59] and Chaudhari et al. [60] investigated the influence of cavity heights on the flow characteristics of SJ experimentally. The cavity heights of their study were $6.3,8.7,11$ and $13.5 \mathrm{~mm}$. The Reynolds Numbers, $\operatorname{Re}_{\bar{U}_{\text {ave }}}$ varied from 412 to 1096 while the stroke ratio, $L_{\bar{U}_{\text {ave }}}$ were ranged between 0.112 and 1.064. They reported that the curves of the averaged velocity (over an oscillating cycle) against the excitation frequency has exhibited two peaks. The first peak corresponds to the natural frequency of the diaphragm while the second peak corresponds to the Helmholtz resonance frequency. When the diaphragm excitation frequency was coinciding with the Helmholtz Resonance frequency, the exit velocity increased with higher cavity height. However, when the two frequencies were not coinciding with each other, the exit velocity was a weak function of the cavity depth.

Lv et al. [54] also investigated the effect of cavity height under the condition where the diaphragm excitation frequency is coinciding with the Helmholtz Resonance frequency, but by a numerical approach. In their study, the cavity heights were $3.44,6.42$ and $7.06 \mathrm{~mm}$, which 
were chosen to match the cavity acoustic Helmholtz resonance frequency with the diaphragm excitation frequency. Hence, the study designated them as cavity acoustic resonance radiuses. They further explained that the fluid inertial action inside the cavity ceases to occur during expulsion and ingestion phase in a smaller cavity depth that corresponds to a higher Helmholtz Resonance frequency. Subsequently, it will lead to higher average blowing velocity at the orifice exit as the cavity height corresponding to the Helmholtz Resonance frequency decreases. This statement is supported by Tang and Zhong [51] which proposed a relation for indicating the cavity height is inversely proportional to the Helmholtz Resonance frequency (as shown in Eq. (11)) as well as the maximum jet peak velocity generated under Helmholtz Resonance condition (as shown in Eq. (12)). In addition, Lv et al. [54] clarified that Eq. (8) was derived based on simplified lumped element model and the fluid inertial action inside the cavity may not be sufficiently inspected as compared to the real Helmholtz Resonance regime. This might be the reason that contributed to the significant discrepancies between the numerical and experimental findings.

\subsubsection{Effect of Cavity Diameter on Jet Velocity}

Jain et al. [53] investigated numerically the effect of cavity diameter $D_{c}$ on the flow characteristics of SJ under two conditions, namely, at constant vibration amplitude and constant swept volume. In their numerical method, only a two-dimensional domain was simulated with the assumption of axisymmetric jet flow behaviour. The diaphragm spanned the whole cross section of the cavity. When the swept volume was held constant and the SJA was not operated near the Helmholtz resonance frequency, the maximum velocity increased gradually from $D_{c}$ $=20$ to $50 \mathrm{~mm}$ before it reached a peak at $D_{c}=60 \mathrm{~mm}$ and decreased afterwards. They explained that at a constant swept volume, when the cavity diameter is smaller and the vibration 
amplitude is higher, the acceleration of the diaphragm would be higher. As such, the flow inside the cavity would have insufficient time to adapt to the rapid vibration motion of the diaphragm. Thus, the exit velocity would be lower. On the other hand, when the cavity diameter is larger, the fluid inside the cavity would fail to reach the orifice exit while the diaphragm begins to move in the direction opposite to the flow motion, which leads to a lower mass flow rate and exit velocity. Hence, they deduced that for a given swept volume, there is an optimum cavity diameter which could produce the highest momentum transfer between the diaphragm and the fluid.

Lv et al. [542] employed a numerical method to investigate the influence of various SJA geometric parameters including the cavity radius on the flow characteristics of SJ. In their study, the cavity radiuses were $15.9,22.5$ and $35.7 \mathrm{~mm}$. These radiuses were chosen to match the cavity acoustic Helmholtz resonance frequency with the diaphragm excitation frequency. Hence, the study designated them as cavity acoustic resonance radiuses. The stroke ratio of the study were $5.535 \leq L_{\bar{U}_{e}} \leq 10.728$. The results depicted that the peak average ejecting velocity at the orifice exit increased with smaller cavity acoustic resonance radius. They explained that a smaller cavity acoustic resonance radius which corresponds to a higher Helmholtz Resonance frequency reduces the inertial action of the fluid in the vicinity of orifice exit. Thus, the expulsion and ingestion at the orifice exit are able to respond efficiently to the diaphragm excitation frequency. This would enhance the maximum jet velocity.

\subsection{Effects of Orifice Edges on the Performance of a Synthetic Jet}

Conventionally, the orifices of most SJAs have sharp edges. In the literature, various comparative studies have been carried out to examine the effects of different edge configurations (i.e., bevel, round and cusp shapes) on the characteristics of the jet velocity. 
Moreover, a few such studies have also found that a rounded orifice edge plays an important role in influencing the effectiveness of the jet. These studies mainly focused on the effect of the orifice edge on the jet velocity characteristics and jet effectiveness, although the mass flow rate and flow behaviour were also mentioned. This section reviews the comparative studies between sharp edges and other orifice edge configurations.

Lee and Goldstein [55] carried out a numerical study to investigate the effect of 2D slotted orifice edges on the flow field of SJ. Three different types of orifice edges, i.e. flat, round, and cusp shapes (Fig. 7) were compared under the constant Reynolds Number, $R e_{\bar{U}_{\text {peak }}}=$ 104.2 and stroke ratio, $L_{\bar{U}_{\text {peak }}}=15.9$ (note that the $R e_{\bar{U}_{\text {peak }}}$ and $L_{\bar{U}_{\text {peak }}}$ are based on slot halfwidth, $\left.w_{\frac{1}{2}}\right)$. Their results showed that the rounded edge case has exhibited a lower peak streamwise velocity (around 20\% lower) than the flat edge case. They explained that the lower value is attributed to the prevention of flow separation in the former, and thus restricts the constriction effect through the slot. In addition, the rounded edge configuration is able to entrain or expulse more fluids during the ingestion and expulsion phases. Hence, it leads to a higher spanwise velocity than the flat edge case. Meanwhile, the cusp-shaped edge case has a similar streamwise velocity development to the flat edge case despite the small changes in both the spanwise velocity and vorticity. 
(a)
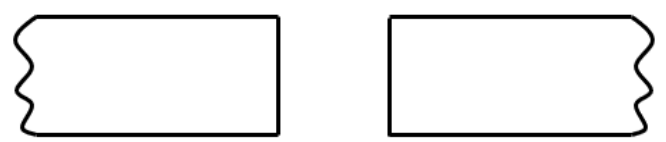

(b)
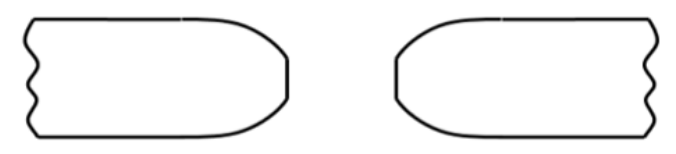

(c)

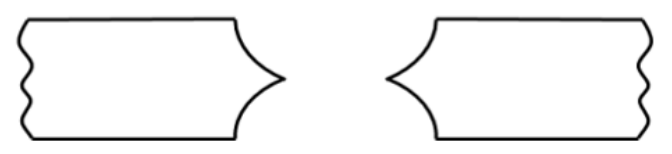

Fig. 7: Diagram of types of orifice edges used by Lee and Goldstein [55] (a) flat (b) round (c) cusp shapes.

Cater and Soria [4] carried out an experimental study to investigate the structure and mean flow quantities of circular SJA. As illustrated in Fig. 8, two types of orifice plates, i.e. blunt-edged and bevel-edged were considered in the study. They used the blunt-edged orifice to obtain the flow visualization results, but have replaced it with the bevel-edged orifice during velocity measurements. The reason was that the bevel-edged orifice can reduce the flow separation at the orifice inner edges during the expulsion stroke. Besides, the bevel-shaped orifice ( $45^{\circ}$ bevel angle on both sides) is able to preserve the symmetrical flow at the orifice during expulsion and ingestion stroke. Their comparative flow visualization results showed that both of the orifice plates had behaved similarly in the development of jet. 
(a)
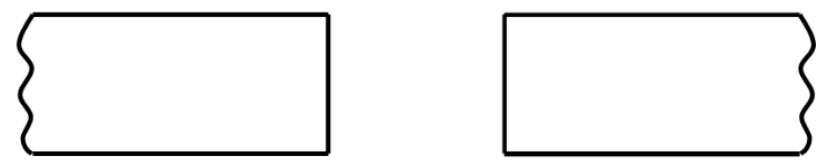

(b)

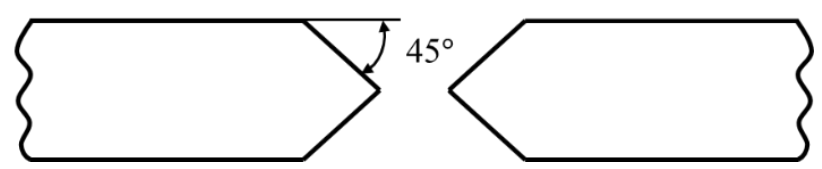

Fig. 8: Orifice plates used by Cater and Soria [4] $(a)$ blunt-edged $(b)$ bevel-edged with $45^{\circ}$ bevel angle on both sides.

Shuster and Smith [61] experimentally investigated the effect of circular orifice edge configurations on the flow field generated from piston-cylinder SJA. Three different types of orifice edges were studied, i.e. straight, beveled and rounded (Fig. 9). For a given Reynolds number, $R e_{\bar{U}_{e}}=2500$, as the stroke ratio increased from 1 to 3 , the coherent rings in the beveled case had propagated faster than the straight case (Fig. 10). Furthermore, the breakdown of vortex rings did not occur in the beveled case during $L_{\bar{U}_{e}}=1$ which was opposed to the straight case. In addition, for increasing Reynolds number, $1000 \leq R e_{\bar{U}_{e}} \leq 5000$, and stroke ratio, $1 \leq L_{\bar{U}_{e}} \leq 3$, the jet exit velocity profiles of the beveled case are more similar to one another at each phase than the straight orifice case (see Fig. 11 for the cases at $R e_{\bar{U}_{e}}=2500$ ). 
(a)
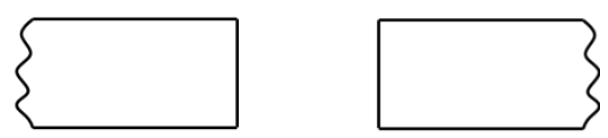

(b)
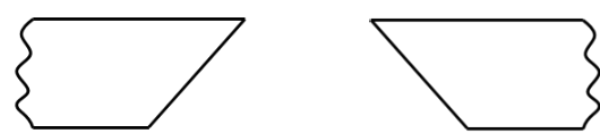

(c)
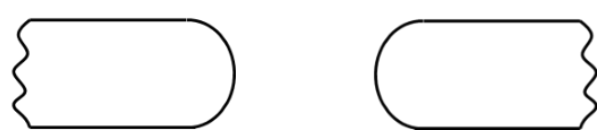

Fig. 9: Diagram of types of orifice edges used by Shuster and Smith [61] (a) straight (b) beveled $(c)$ rounded. 
(a)
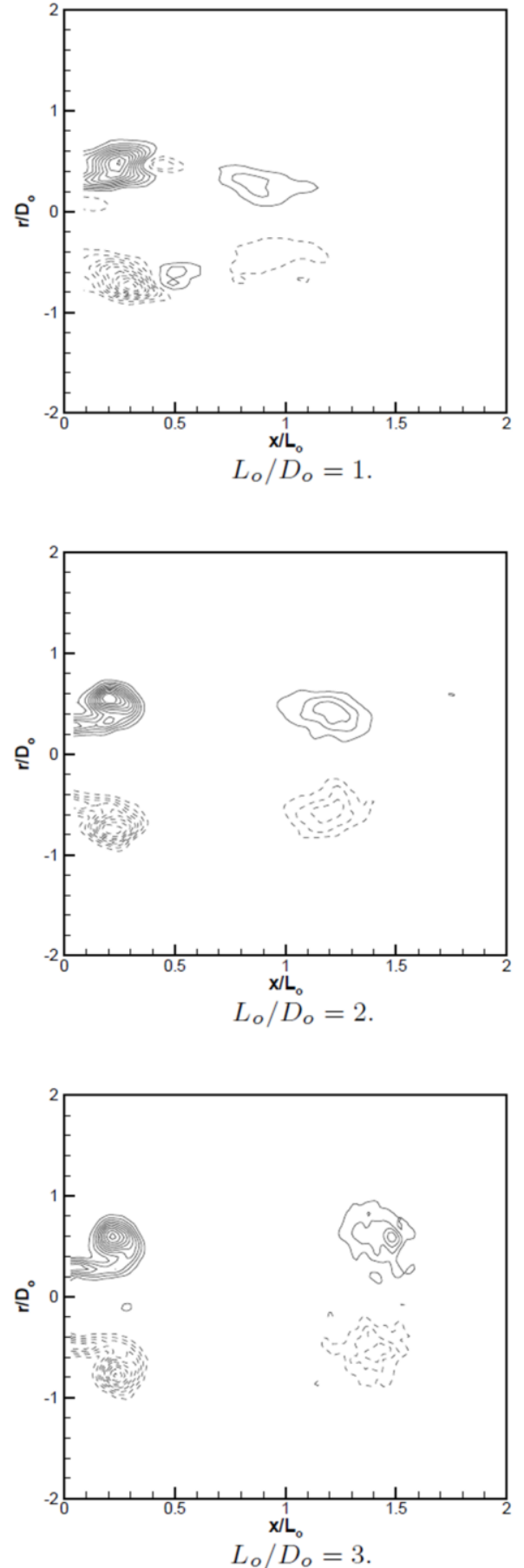

(b)
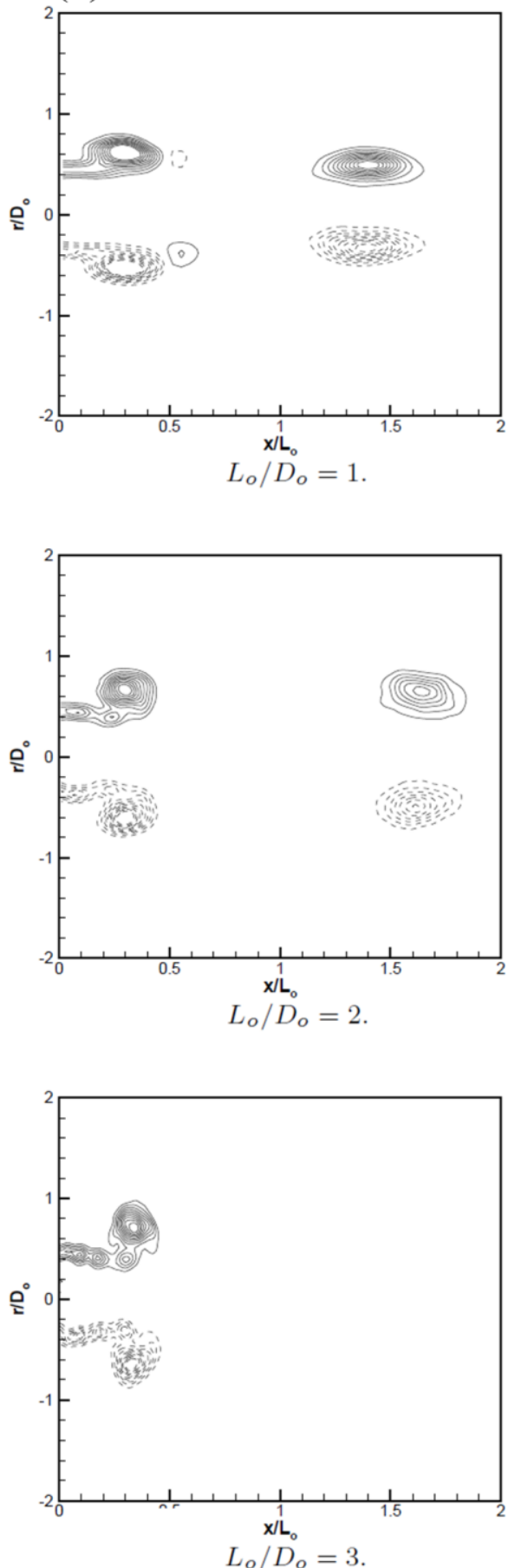

Fig. 10: Effect of orifice edge configuration at $R e_{\bar{U}_{e}}=2500$; Contours of vorticity for the (a) straight orifice and (b) rounded orifice; Reproduced with permission from J. M. Shuster and D. R. Smith, "A Study of the Formation and Scaling of a Synthetic Jet," AIAA, pp. 1-15, 2004. Copyright 2004 Jennifer M. Shuster. 
(a)
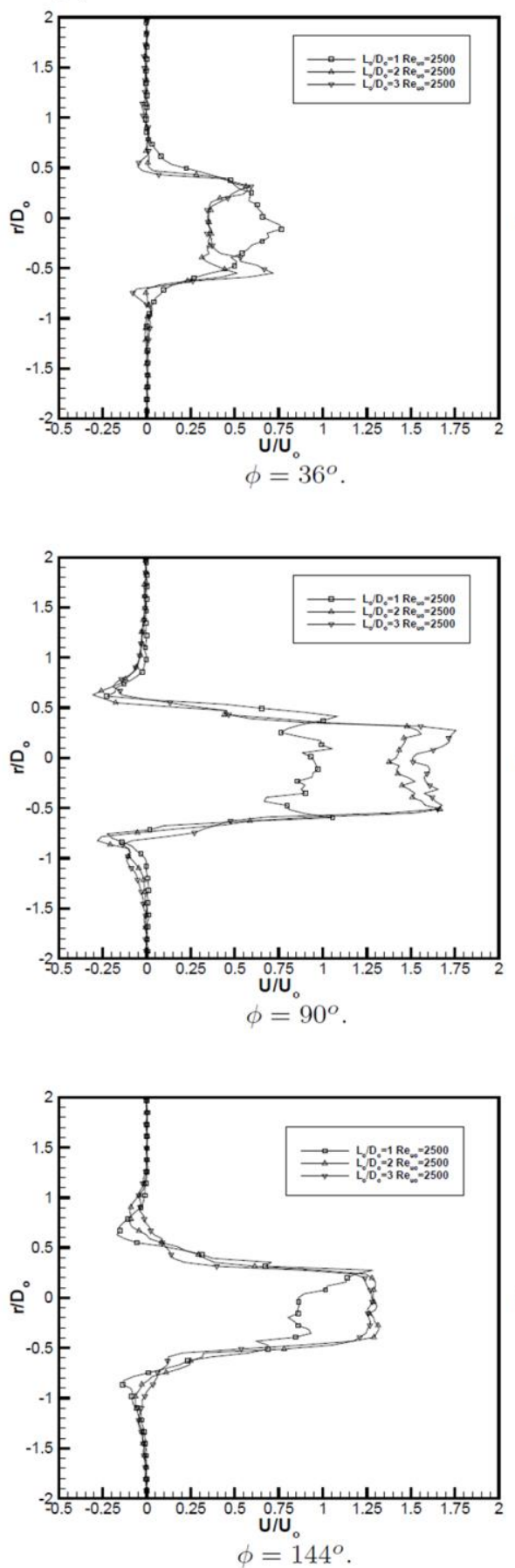

(b)
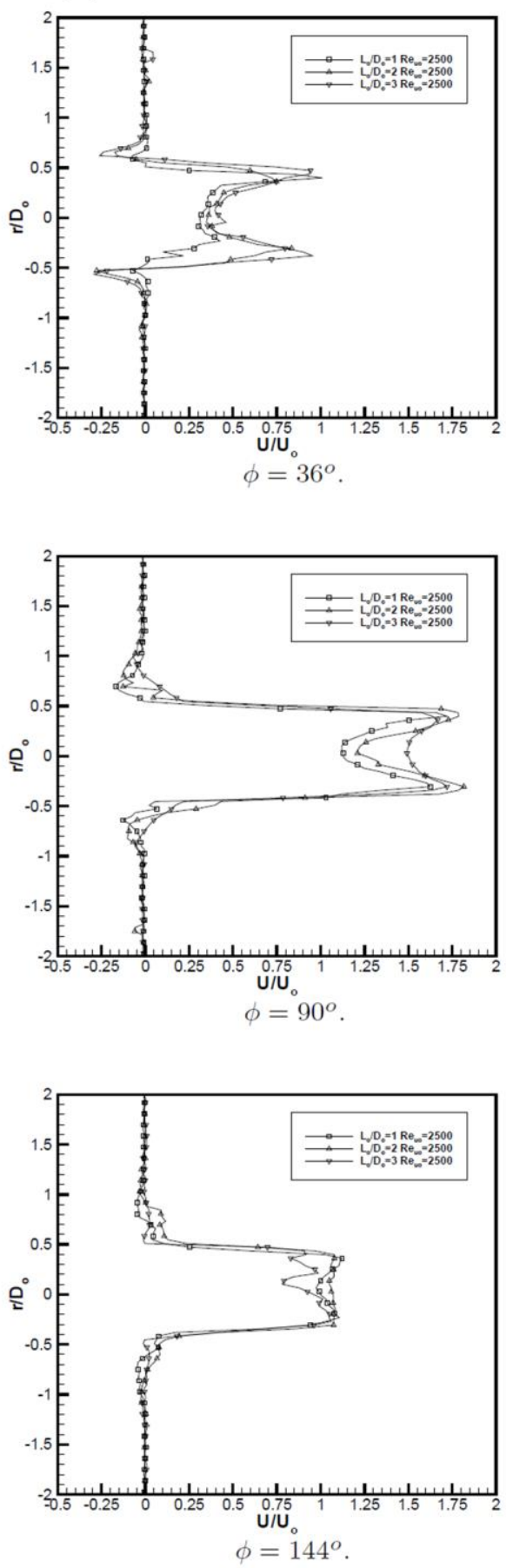

Fig. 11: Effect of orifice edge configuration at $R e_{\bar{U}_{e}}=2500$; Exit velocity profiles for the (a) straight orifice and (b) bevelled orifice; Reproduced with permission from J. M. Shuster and D. R. Smith, "A Study of the Formation and Scaling of a Synthetic Jet," AIAA, pp. 1-15, 2004. Copyright 2004 Jennifer M. Shuster. 
The subsequent numerical study of Jain et al. [53] has examined the influence of sharp and rounded orifice edge configurations on the performance of SJA. A normal circular orifice (with sharp inner and outer edges) and three different rounded orifice cases (i.e. rounded at the inner (cavity side), outer (orifice exit side), and both sides of the orifice) were studied. The radius of the edges was held constant at $r=0.5 \mathrm{~mm}$ for all the cases. Their results revealed that the peak velocity induced by the case of orifice with both sides rounded is $25 \%$ lower than the normal orifice despite the similar trend in velocity profile. The results agreed well with the previous numerical work [55]. They clarified that the rounded edges reduce the flow separation at the entrance as well as the constriction effect in the orifice duct, which is similar to the results obtained by Lee and Goldstein [55]. They further explained that the vortex formation inside the orifice diminishes as more fluid can be drawn from the rounded edges. Besides, their results showed that inner-side-rounded case has produced a lower velocity than the normal orifice case. Whilst, the outer-side-rounded case has generated the same velocity as the normal orifice case. However, when either side of the orifice is rounded, higher velocity is produced than the bothsides-rounded case. Highest mass flow rate can be obtained from the case with rounded both sides, followed by the case with rounded outer side and normal orifice.

Jain et al. [53] further investigated the effect of bevel-shaped orifice on the characteristics of a SJ flow. The nozzle angle was varied from $0^{\circ}$ to $30^{\circ}$ (i.e. $0^{\circ}, 5^{\circ}, 10^{\circ}, 15^{\circ}$, $20^{\circ}$, and $30^{\circ}$ ). A fixed diameter of $3 \mathrm{~mm}$ was used at the centre of the converging section (Fig. 12). The Reynolds number based on the orifice exit diameter was varied between 903 and 1494. In their numerical study, the maximum exit velocities at $1 \mathrm{~mm}$ away from the orifice exit were obtained for all the cases. Their results depicted that when the nozzle angle was smaller than $15^{\circ}$, the maximum exit velocity was lower than the normal orifice (i.e. $0^{\circ}$ ). This is attributed to the increment of distance travelled by the fluid along the nozzle wall which leads to higher flow resistance. However, the maximum exit velocity was higher than the case with normal 
orifice at nozzle angle larger than $15^{\circ}$. They explained that the results are due to flow separation at the nozzle wall and is accompanied by the vortex formation within the length of the nozzle during ingestion stroke. Therefore, the flow resistance induced by the nozzle wall decreases and leads to higher maximum exit velocity. Besides, their results showed that the $15^{\circ}$ nozzle angle case had produced the highest mass flow rate, which was 5\% higher than the value obtained from the normal orifice case. Moreover, the mass flow rates produced at other nozzle angles (i.e. smaller or larger than $15^{\circ}$ ) were lower than the normal orifice case. They explained that as the nozzle angle increases further after $15^{\circ}$, the orifice exit diameter becomes even smaller as compared to the normal orifice. Thus, resulted in a lower mass flow rate and higher maximum jet exit velocity.

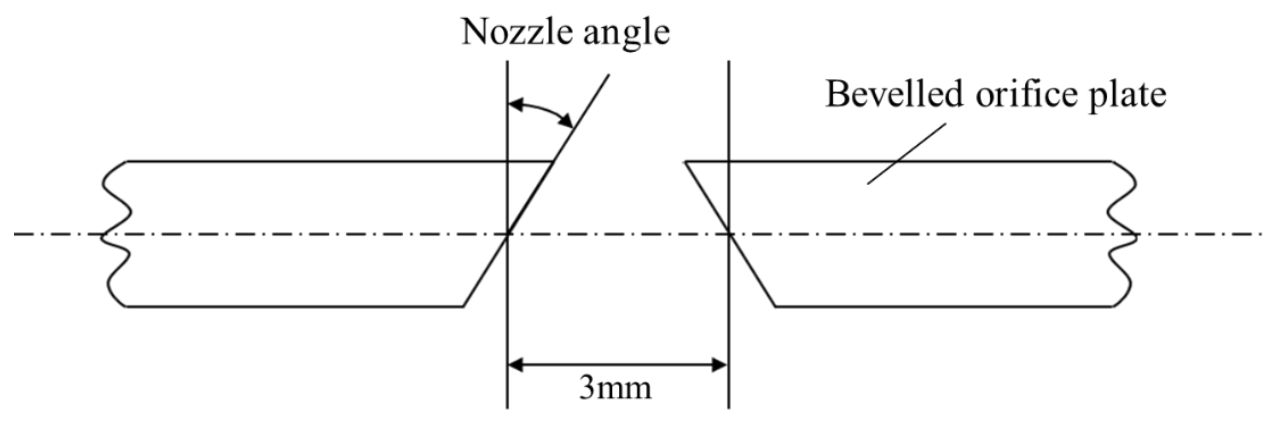

Fig. 12: Bevel-shaped orifice used by Jain et al. [53].

Smith and Swift [62] investigated the effects of rounded edges on the oscillating flow from a $2 \mathrm{D}$ channel into an infinity medium. They conducted the experiment by using channel plate with different outer edges radii, i.e. $r=6.4,12.7$ and $25.4 \mathrm{~mm}$. The time-averaged pressure difference and acoustic power dissipation were measured between two locations corresponding to: (a) inside the orifice duct, (b) at far downstream where the velocity becomes negligible. Both of the quantities were normalized by the dimensionless edge radius, $\frac{r}{w}$ (normalized by variable slot width, $w$ ) under constant stroke ratio, $L_{\bar{U}_{\text {peak }}}=30$, and two 
different Reynolds Numbers, $R e_{\bar{U}_{\text {peak }}}$, i.e. 14432 and 28892 (note that the Reynolds number and stroke ratio are derived as $R e_{\bar{U}_{p e a k}}=\frac{\bar{U}_{e} w}{v}$ and $L_{\bar{U}_{p e a k}}=\frac{\bar{U}_{e}}{f w}$, respectively). Note that $R e_{\bar{U}_{p e a k}}$ is calculated based on $R e_{\delta}=658$ and 931 by using Eq. (13) proposed by Fugal et al. [69]. The results showed that the normalized time-averaged pressure difference had decreased with rounder orifice edge despite the difference in $R e_{\bar{U}_{\text {peak }}}$. This is attributed to larger expansion of ejecting flow at the orifice exit. Hence, it leads to smaller pressure difference between locations (a) and (b) as the edge radius increases. Meanwhile, the $\frac{r}{w}$ shows a minor effect on normalized acoustic power dissipation and leads to an inconsistent trend under different $R e_{\bar{U}_{\text {peak }}}$. In their study, the ratio of normalized time-averaged pressure difference to normalized acoustic power dissipation is defined as the jet effectiveness. The effectiveness had declined when the $\frac{r}{w}$ increased since the parameter is proportional to normalized timeaveraged pressure difference without being affected by the weak relation in normalized acoustic power dissipation. Hence, increasing the edge radius tends to reduce the effectiveness of oscillating flow in terms of time-averaged pressure difference.

$R e_{\bar{U}_{p e a k}}=\frac{R e_{\delta}^{2}}{L_{o} / w}$

where, $R e_{\delta}=$ Reynolds number based on viscous penetration depth, $R e_{\delta}=\bar{U}_{\text {peak }} \frac{\sqrt{\frac{v T}{\pi}}}{v}, L_{o}=$ stroke length, and $w=$ slot width.

Fugal et al. [63] extended the above experimental work and investigated the effect of sharp and rounded orifice outer edges by using computational approach. Two dimensionless edges radii, i.e. $\frac{r}{w}=0$ and $\frac{r}{w}=1$ were used to represent the SJAs with sharp and rounded outer edges. For a given Reynolds Number, $R e_{\bar{U}_{e}}=33333$ and a range of stroke ratio, $2 \leq L_{\bar{U}_{e}} \leq$ 
30 (note that the Reynolds number and stroke ratio are derived as $R e_{\bar{U}_{e}}=\frac{\bar{U}_{e} w}{v}$ and $L_{\bar{U}_{e}}=\frac{\bar{U}_{e}}{f w}$, respectively in this study), the case with sharp outer edges $\left(\frac{r}{w}=0\right)$ required a higher acoustic power magnitude than the case with rounded configuration $\left(\frac{r}{w}=1\right)$ during jet formation. The results are well validated with the experimental work of Smith and Swift [62]. They attributed the increment of acoustic power in the sharp outer edges case to the losses that occurred at the sharp exit during ingestion phase. A similar outcome was observed in which, at $R e_{\bar{U}_{e}}=33333$ and $L_{\bar{U}_{e}}$ ranges between 2 and 30, the sharp outer edges orifice had induced a higher normalized final momentum flux than its rounded counterpart. This is due to the fact that the ejected fluid decelerates in the vicinity of rounded orifice exit, hence resulting in lower momentum flux. In their study, the jet effectiveness was defined as the ratio of normalized final momentum flux to the normalized acoustic power dissipation. The jet effectiveness of the rounded outer edges case was higher than the sharp outer edges case as the former requires relatively lower acoustic power.

Nani and Smith [64] conducted an experiment to examine the influence of orifice inner edges radius on the efficiency of SJ. Four inner edges radii, i.e. $r=0,3.969,6.35$ and 12.7 $\mathrm{mm}$ (corresponding to $0 D_{o}, 0.3125 D_{o}, 0.5 D_{o}$ and $1 D_{o}$ respectively) were compared while sharp edges were used for the outer orifice. Besides, all cases were carried out at constant $\operatorname{Re}_{\bar{U}_{e}}=$ 35000 and three different $L_{\bar{U}_{e}}=10,15$ and 20. The result tendency of the normalized acoustic power was different than the one obtained from the previous experimental and numerical works. For a fixed $L_{\bar{U}_{e}}$, the normalized input acoustic power had first decreased when the inner edges radii increased from $r=0 D_{o}$ to $0.5 D_{o}$, then it increased significantly at $r=1 D_{o}$. This is attributed to the absence of straight wall portion of the orifice duct in the case of $r=1 D_{o}$ as the flow ceases to attach to the inner edges radius while entering the cavity. Therefore, it leads to higher suction losses and acoustic power. Furthermore, this phenomena does not appear in 
the previous studies by Smith and Swift [62] and Fugal et al. [63] as both of them had used a long orifice flow channel. As for the normalized momentum flux, the result had shown weak dependency on the inner edges radius under constant $R e_{\bar{U}_{e}}$ and $L_{\bar{U}_{e}}$.

In summary, a number of comparative studies examined the effects of orifice edge configurations. The shapes considered comprised the straight edge, a number of variations of the rounded edge (i.e. rounded on the inner side, the outer side, and on both the inner and outer sides, as well as with varying radii), the bevelled edge with varying nozzle angles, and the cusp-shaped edge. The results showed that the orifice edge configuration can have a significant impact on the characteristics of the vortex ring, including the peak velocity, the mass flow rate, the jet effectiveness, the momentum flux, and the acoustic power. Therefore, it is possible to configure the orifice edge to obtain SJ properties that are tailored to the needs of specific applications.

\subsection{Concluding Remarks}

In general, a synthetic jet issuing into a quiescent fluid has a flow field with three different flow regimes: near-field region, transitional region, and far-field region. In the nearfield region, the flow structure is developed based on the formation and conveyance of discrete vortex rings under time-periodic reverse flow conditions. For the transitional region, the vortex rings entrain the ambient fluid into the jet before breaking into smaller eddies in the far-field region. The characteristics and performance of synthetic jets are determined by the actuator parameters, geometrical parameters and fluid parameters. The effects of the geometrical parameters on synthetic jets in quiescent flows were discussed and reviewed systematically. 
Jets produced by non-circular orifices are susceptible to the phenomenon of axis switching, and thus, will exhibit a higher decay rate. In addition, as the aspect ratio of the rectangular orifice increases, the axis-switching behaviour has been found to become more vigorous, and tends to occur further downstream. An orifice with a higher aspect ratio will also exhibit a higher jet exit velocity. However, the time-averaged velocity of the jet is not linearly proportional to the aspect ratio when the excitation frequency corresponds to the Helmholtz resonance frequency. Instead, there is an optimal aspect ratio in which the maximum timeaveraged velocity can be obtained.

Studies on the effects of the orifice depth suggest that an optimum orifice depth exists, by which the maximum velocity can be attained. In addition, when the orifice depth is smaller than the orifice size, a lower blowing velocity will be produced due to the absence of a constriction effect. However, at a low Helmholtz resonance frequency, the orifice depth has been found to have no significant influence on the blowing velocity.

The effect of cavity height has been found to vary from one SJA type to another. While some studies reported an inversely proportional relationship between the jet velocity and cavity height, others found a weak relationship between the two variables. Furthermore, an experimental study reported that when the diaphragm excitation frequency coincided with the Helmholtz resonance frequency, the exit velocity was found to increase with the cavity height. However, under the desynchronized condition, the two variables were weakly related.

For a given actuation amplitude, the maximum jet velocity is directly proportional to the cavity diameter. However, for a given swept volume, there is an optimal cavity diameter by which the maximum jet velocity can be attained. Besides, when the excitation frequency is synchronized with the Helmholtz resonance frequency, the maximum average blowing velocity has been found to be inversely proportional to the cavity diameter. 
Despite decades of research, the contradictory findings pertaining to the effects of the aspect ratio of rectangular jets on the velocity decay rate suggest that there is a need for further investigation. In particular, more levels of the aspect ratio should be inspected at distinct stroke ratio ranges to allow for a more comprehensive analysis of the effects.

\section{ACKNOWLEDGEMENT}

This project is supported by Universiti Teknikal Malaysia Melaka (UTeM) and Ministry of Higher Education under FRGS/2018/FKM-CARe/F00375.

\section{REFERENCES}

[1] N. Mane, P., Mossi, K., Rostami, A., Bryant, R. and Castro, "Piezoelectric Actuators as Synthetic Jets: Cavity Dimension Effects,” J. Intell. Mater. Syst. Struct., vol. 18, no. 11, pp. 1175-1190, 2007.

[2] M. Chaudhari, B. Puranik, and A. Agrawal, "Heat transfer characteristics of synthetic jet impingement cooling," Int. J. Heat Mass Transf., vol. 53, no. 5-6, pp. 1057-1069, 2010 .

[3] G. Paolillo, C. S. Greco, and G. Cardone, "The evolution of quadruple synthetic jets," Exp. Therm. Fluid Sci., no. August, 2017.

[4] J. E. Cater and J. Soria, "The evolution of round zero-net-mass-flux jets," J. Fluid Mech., vol. 472, pp. 167-200, 2002.

[5] G. Maria, D. Cicca, and G. Iuso, "On the near field of an axisymmetric synthetic jet," Fluid Dyn. Res., vol. 39, pp. 673-693, 2007. 
[6] T. Murugan, M. Deyashi, S. Dey, S. C. Rana, and P. K. Chatterjee, "Recent Developments on Synthetic Jets,” Def. Sci. J., vol. 66, no. 5, pp. 489-498, 2016.

[7] H. Tang and S. Zhong, Vortex Rings and Jets, vol. 111. 2015.

[8] A. Glezer and Michael Amitay, "Synthetic Jets," Annu. Rev. Fluid Mech., 2002.

[9] X. Xia and K. Mohseni, “Axisymmetric Synthetic Jets : Modeling of the Far-field Momentum Flux," 53rd AIAA Aerosp. Sci. Meet. AIAA, no. January, pp. 1-14, 2015.

[10] Y. Chen, S. Liang, K. Anug, A. Glezer, and J. Jagoda, "Enhanced Mixing in a Simulated Combustor Using Synthetic Jet Actuators," 37th AIAA Aerosp. Sci. Meet. Exhib., no. c, p. AIAA-99-0449, 1999.

[11] M. Amitay, D. R. Smith, V. Kibens, D. E. Parekh, and A. Glezer, “Aerodynamic Flow Control over an Unconventional Airfoil Using Synthetic Jet Actuators," AIAA J., vol. 39, no. 3, pp. 361-370, 2001.

[12] M. Amitay and F. Cannelle, "Evolution of finite span synthetic jets," Phys. Fluids, vol. 18, no. 5, 2006.

[13] J. D. Vasile and M. Amitay, "Interaction of a finite span synthetic jet near the tip of a sweptback wing," Phys. Fluids, vol. 27, p. 27, 2015.

[14] Y. Elimelech, J. Vasile, and M. Amitay, "Secondary flow structures due to interaction between a finite-span synthetic jet and a 3-D cross flow," Phys. Fluids, vol. 23, no. 9, 2011.

[15] T. Van Buren, C. M. Leong, E. Whalen, and M. Amitay, "Impact of orifice orientation on a finite-span synthetic jet interaction with a crossflow," Phys. Fluids, vol. 28, no. 3, pp. 1-20, 2016. 
[16] M. Jabbal and S. Zhong, "Particle image velocimetry measurements of the interaction of synthetic jets with a zero-pressure gradient laminar boundary layer," Phys. Fluids, vol. 22, no. 6, pp. 1-17, 2010.

[17] W. Zhang and R. Samtaney, "A direct numerical simulation investigation of the synthetic jet frequency effects on separation control of low-Re flow past an airfoil," Phys. Fluids, vol. 27, no. 5, p. 22, 2015.

[18] K. Taylor and M. Amitay, "Dynamic stall process on a finite span model and its control via synthetic jet actuators," Phys. Fluids, vol. 27, p. 26, 2015.

[19] Y. Xu, Z. Y. Li, J. J. Wang, and L. J. Yang, “On the interaction between turbulent vortex rings of a synthetic jet and porous walls," Phys. Fluids, vol. 31, p. 16, 2019.

[20] T. Van Buren, M. Beyar, C. M. Leong, and M. Amitay, “Three-dimensional interaction of a finite-span synthetic jet in a crossflow," Phys. Fluids, vol. 28, p. 18, 2016.

[21] C. M. Crispo, C. S. Greco, and G. Cardone, "Convective heat transfer in circular and chevron impinging synthetic jets," Int. J. Heat Mass Transf., vol. 126, pp. 969-979, 2018 .

[22] R. Mahalingam and A. Glezer, "Design and Thermal Characteristics of a Synthetic Jet Ejector Heat Sink,” J. Electron. Packag., vol. 127, no. 2, p. 172, 2005.

[23] G. Paolillo, C. S. Greco, and G. Cardone, "Impingement heat transfer of quadruple synthetic jets," Int. J. Heat Mass Transf., vol. 135, pp. 1192-1206, 2019.

[24] A. Pavlova and M. Amitay, "Electronic Cooling Using Synthetic Jet Impingement," $J$. Heat Transfer, vol. 128, no. 9, p. 897, 2006. 
[25] B. L. Smith and A. Glezer, "Jet vectoring using synthetic jets," J. Fluid Mech., vol. 458, pp. 1-34, 2002.

[26] B. L. Smith and A. Glezer, "Vectoring of Adjacent Synthetic Jets," AIAA J., vol. 43, no. 10, pp. 2117-2124, 2005.

[27] I. H. Ibrahim and M. Skote, "Simulations of the linear plasma synthetic jet actuator utilizing a modified Suzen-Huang model," Phys. Fluids, vol. 24, no. 11, pp. 1-19, 2012.

[28] B. Vukasinovic, D. Brzozowski, and A. Glezer, "Fluidic Control of Separation Over a Hemispherical Turret,” AIAA J., vol. 47, no. 9, pp. 2212-2222, 2009.

[29] B. Vukasinovic, A. Glezer, S. Gordeyev, E. Jumper, and V. Kibens, "Fluidic control of a turret wake: Aerodynamic and aero-optical effects," AIAA J., vol. 48, no. 8, pp. 1686-1699, 2010.

[30] L. N. Cattafesta, Q. Song, D. R. Williams, C. W. Rowley, and F. S. Alvi, "Progress in Aerospace Sciences Active control of flow-induced cavity oscillations," Prog. Aerosp. Sci., vol. 44, pp. 479-502, 2008.

[31] V. Narayanaswamy, L. L. Raja, and N. T. Clemens, "Control of unsteadiness of a shock wave/turbulent boundary layer interaction by using a pulsed-plasma-jet actuator," Phys. Fluids, vol. 24, no. 7, pp. 1-22, 2012.

[32] A. Glezer, "The formation of vortex rings," Phys. Fluids, vol. 31, no. 12, pp. 3532$3542,1988$.

[33] B. L. Smith and A. Glezer, "The formation and evolution of synthetic jets," Phys. Fluids, vol. 10, no. 9, pp. 2281-2297, 1998. 
[34] Y. Utturkar, R. Holman, R. Mittal, B. Carroll, M. Sheplak, and L. Cattafesta, “A Jet Formation Criterion for Synthetic Jet Actuators," 41st Aerosp. Sci. Meet. Exhib., no. January, 2003.

[35] R. Holman, Y. Utturkar, R. Mittal, B. L. Smith, and L. Cattafesta, "Formation Criterion for Synthetic Jets,” AIAA J., vol. 43, no. 10, pp. 2110-2116, 2005.

[36] Z. Panfeng, W. Jinjun, and F. Lihao, "Review of zero-net-mass-flux jet and its application in separation flow control," Sci. China Ser. E Technol. Sci., vol. 51, no. 10425207, pp. 1315-1344, 2008.

[37] K. Mohseni and R. Mittal, Synthetic jets Fundamentals and Applications. CRC Press, Taylor \& Francis Group, 2015.

[38] F. Grinstein, "Vortex dynamics and entrainment in regular free jets," J. Fluid Mech., vol. 437, no. August, pp. 69-101, 2001.

[39] C. Ho and E. Gutmark, "Vortex induction and mass entrainment in a small-aspect-ratio elliptic jet,” J. Fluid Mech., vol. 179, 1987.

[40] H. S. Husain and K. M. F. Hussain, "Controlled excitation of elliptic jets," Phys. Fluids, vol. 26, no. 10, p. 2763, 1983.

[41] L. Garcillan, S. Zhong, Z. Pokusevski, and N. Wood, “A PIV Study of Synthetic Jets with Different Orifice Shape and Orientation,” 2nd AIAA Flow Control Conf., pp. 1$13,2004$.

[42] L. Oren, E. Gutmark, S. Muragappan, and S. Khosla, "Flow Characteristics of Non Circular Synthetic Jets," AIAA 2009-1309, p. 2009, 2009.

[43] A. Crook, W. J. Crowther, and N. J. Wood, “A Parametric Study of a Synthetic Jet in 
Quiscent Conditions," 9th Int. Symp. Flow Vis., pp. 1-7, 2000.

[44] B. Ravi, R. Mittal, and F. M. Najjar, "Study of Three-Dimensional Synthetic Jet Flowfields Using Direct Numerical Simulation.," AIAA Aerosp. Sci. Meet., no. January, pp. 1-10, 2004.

[45] T. Van Buren, E. Whalen, and M. Amitay, "Vortex formation of a finite-span synthetic jet : effect of rectangular orifice geometry," J. Fluid Mech., vol. 745, pp. 180-207, 2014.

[46] Y. Kim and K. P. Garry, "Optimization of a Rectangular Orifice Synthetic Jet Generator,"3rd AIAA Flow Control Conf., no. June, pp. 1-9, 2006.

[47] G. Krishnan and K. Mohseni, "An Experimental and Analytical Investigation of Rectangular Synthetic Jets,” J. Fluids Eng., vol. 131, p. 11, 2017.

[48] T. van Buren, E. Whalen, and M. Amitay, "Vortex formation of a finite-span synthetic jet: High Reynolds numbers," Phys. Fluids, vol. 26, no. 1, 2014.

[49] L. Wang, L.-H. Feng, J.-J. Wang, and T. Li, "Parameter influence on the evolution of low-aspect-ratio rectangular synthetic jets," J. Vis., 2017.

[50] Q. Gallas et al., "Lumped Element Modeling of Piezoelectric-Driven Synthetic Jet Actuators," AIAA, vol. 41, no. 2, pp. 1-10, 2002.

[51] H. Tang and S. Zhong, "Modelling of the Characteristics of Synthetic Jet Actuators," 35th AIAA Fluid Dyn. Conf. Exhib., no. June, pp. 1-13, 2005.

[52] M. Jabbal, H. Tang, and S. Zhong, "The effect of geometry on the performance of synthetic jet actuators,” 25TH Int. Congr. Aeronaut. Sci., vol. 25, no. March 2014, 2006. 
[53] M. Jain, B. Puranik, and A. Agrawal, "A numerical investigation of effects of cavity and orifice parameters on the characteristics of a synthetic jet flow," Sensors Actuators A. Phys., vol. 165, pp. 351-366, 2011.

[54] L. Yuan-wei, Z. Jing-zhou, S. Yong, and T. Xiao-ming, "Sensors and Actuators A : Physical Numerical investigation for effects of actuator parameters and excitation frequencies on synthetic jet fluidic characteristics," Sensors Actuators A. Phys., vol. 219, pp. 100-111, 2014.

[55] C. Y. Lee and D. B. Goldstein, "Two-Dimensional Synthetic Jet Simulation," AIAA J., vol. 40, no. 3, 2002.

[56] S. G. Mallinson, G. Hong, and J. A. Reizes, "Some Characteristics of Synthetic Jets," 30th AIAA Fluid Dyn. Conf., 1999.

[57] P. Mane, "Experimental Design and Analysis of Piezoelectric Synthetic Jets in Quiescent Air,” Master dissertation, Virginia Commonwealth University, 2005.

[58] D. P. Rizzetta, M. R. Visbal, M. J. Stanek, U. S. A. Force, W. Patterson, and A. Force, "Numerical Investigation of Synthetic-Jet Flow elds Introduction," AIAA, vol. 37, no. $8,1999$.

[59] M. Chaudhari, G. Verma, A. Baramade, B. Puranik, and A. Agrawal, "Near-field measurements and cavity design for synthetic jet," 38th AIAA Fluid Dyn. Conf. Exhib., no. June, pp. 1-12, 2008.

[60] M. Chaudhari, G. Verma, B. Puranik, and A. Agrawal, "Frequency response of a synthetic jet cavity," Exp. Therm. Fluid Sci., vol. 33, no. 3, pp. 439-448, 2009.

[61] J. M. Shuster and D. R. Smith, "A Study of the Formation and Scaling of a Synthetic 
Jet," AIAA, pp. 1-15, 2004.

[62] B. L. Smith and G. W. Swift, "Power dissipation and time-averaged pressure in oscillating flow through a sudden area change," J. Acoust. Soc. Am., vol. 113, no. 5, pp. 2455-2463, 2003.

[63] S. R. Fugal, B. L. Smith, and R. E. Spall, "Displacement amplitude scaling of a twodimensional synthetic jet," Phys. Fluids, vol. 17, p. 10, 2005.

[64] D. J. Nani and B. L. Smith, "Effect of orifice inner lip radius on synthetic jet efficiency," Phys. Fluids, vol. 24, pp. 1-16, 2012.

\section{LIST OF TABLE CAPTIONS}

Table 1: Governing parameters of synthetic jet

\section{LIST OF FIGURE CAPTIONS}

Fig. 1: Labelling of geometrical parameters of synthetic jet

Fig. 2: Experimental measurement plane (a) along and $(b)$ across the long axis of the slotted orifice (Top); Schematic of axis switching (Bottom).

Fig. 3: Velocity variations along the centerline of synthetic jets from the (a) circular orifice and (b) to (c) rectangular orifices; (b) $\mathrm{AR}=5$, (c) $\mathrm{AR}=10$, and (d) $\mathrm{AR}=16$. In all the cases the peak to peak diaphragm displacement is $0.65 \mathrm{~mm}$; Reproduced with permission from $\mathrm{L}$. Garcillan, S. Zhong, Z. Pokusevski, and N. Wood, “A PIV Study of Synthetic Jets with 
Different Orifice Shape and Orientation," 2nd AIAA Flow Control Conf., pp. 1-13, 2004. Copyright 2004 University of Manchester.

Fig. 4: Variation of average velocity with excitation frequency for different cavity depths and orifice diameters; The orifice diameters are (a) $3 \mathrm{~mm}$, (b) $5 \mathrm{~mm}$, and (c) $8 \mathrm{~mm}$; Reproduced with permission from M. Chaudhari, G. Verma, B. Puranik, and A. Agrawal, "Frequency response of a synthetic jet cavity," Exp. Therm. Fluid Sci., vol. 33, no. 3, pp. 439-448, 2009. Copyright 2008 Elsevier Inc.

Fig. 5: Flow ejected from orifice exit for $a) h=5 \mathrm{~mm}, b) h=2.5 \mathrm{~mm}$, and c) $h=1.25 \mathrm{~mm}$, which are corresponding to $1 D_{o}, 0.5 D_{o}$, and $0.25 D_{o}$ respectively.

Fig. 6: Effect of cavity height on the velocity at the orifice exit; Reproduced with permission from M. Jain, B. Puranik, and A. Agrawal, “A numerical investigation of effects of cavity and orifice parameters on the characteristics of a synthetic jet flow," Sensors Actuators A. Phys., vol. 165, pp. 351-366, 2011. Copyright 2010 Elsevier B.V.

Fig. 7: Diagram of types of orifice edges used by Lee and Goldstein [55] (a) flat (b) round (c) cusp shapes.

Fig. 8: Orifice plates used by Cater and Soria [4] (a) blunt-edged (b) bevel-edged with $45^{\circ}$ bevel angle on both sides.

Fig. 9: Diagram of types of orifice edges used by Shuster and Smith [61] (a) straight $(b)$ beveled $(c)$ rounded.

Fig. 10: Effect of orifice edge configuration at $R e_{\bar{U}_{e}}=2500$; Contours of vorticity for the (a) straight orifice and (b) rounded orifice; Reproduced with permission from J. M. Shuster and D. R. Smith, "A Study of the Formation and Scaling of a Synthetic Jet," AIAA, pp. 1-15, 2004. Copyright 2004 Jennifer M. Shuster. 
Fig. 11: Effect of orifice edge configuration at $R e_{\bar{U}_{e}}=2500$; Exit velocity profiles for the (a) straight orifice and (b) bevelled orifice; Reproduced with permission from J. M. Shuster and D. R. Smith, "A Study of the Formation and Scaling of a Synthetic Jet," AIAA, pp. 1-15, 2004. Copyright 2004 Jennifer M. Shuster.

Fig. 12: Bevel-shaped orifice used by Jain et al. [53].

\section{NOMENCLATURE}

$D_{0} \quad$ Orifice diameter

$D_{c} \quad$ Cavity diameter

H Cavity depth

$h \quad$ Orifice depth

$r_{o} \quad$ Orifice radius

$r \quad$ Edge radius

$V_{c} \quad$ Volume of cavity

w Slot width

$L_{o} \quad$ Stroke Length

$L_{j} \quad$ Jet area based length scale, $(w \times l)^{\frac{1}{2}}$

$R e_{\bar{U}_{e}} \quad$ Reynolds number based on time-averaged velocity induced by SJA during expulsion stoke, $R e_{\bar{U}_{e}}=\frac{\bar{U}_{e} D_{0}}{v}$

$R e_{\bar{U}_{\text {ave }}} \quad$ Reynolds number based on time-averaged velocity induced by SJA during one cycle,

$R e_{\bar{U}_{\text {ave }}}=\frac{\bar{U}_{\text {ave }} D_{0}}{v}$

$R e_{\bar{U}_{r e f}} \quad$ Reynolds number based on reference velocity, $R e_{\bar{U}_{r e f}}=\frac{\bar{U}_{r e f} D_{o}}{v}$

$R e_{\bar{U}_{\text {peak }}} \quad$ Reynolds number based on peak average velocity, $R e_{\bar{U}_{\text {peak }}}=\frac{\bar{U}_{\text {peak }} D_{o}}{v}$ 


$$
\begin{array}{ll}
R e_{\bar{U}_{\text {mean }}} & \text { Reynolds number based on mean velocity, } R e_{\bar{U}_{\text {mean }}}=\frac{\bar{U}_{\text {mean }} D_{O}}{v} \\
R e_{J} & \text { Reynolds number based on jet area based length scale, } R e_{J}=\frac{\bar{V}_{j} L_{j}}{v} \\
w_{\frac{1}{2}} & \text { Slot half-width } \\
l & \text { Length of rectangular orifice (slot orifice) } \\
f & \text { Operating frequency of oscillating diaphragm } \\
T & \text { Time period of excitation cycle } \\
\Delta & \text { Peak to peak displacement of oscillating diaphragm }
\end{array}
$$

AR Aspect ratio

HRF Helmholtz Resonance frequency

$\bar{U}_{e} \quad$ Time-averaged velocity induced by SJA during expulsion stroke

$\bar{U}_{\text {ave }} \quad$ Time-averaged velocity induced by SJA during one cycle

$R e_{\bar{U}_{o}} \quad$ Reynolds number based on velocity scale with momentum flux, $R e_{\bar{U}_{o}}=$ $\frac{\sqrt{2} L f w}{v}$

$R e_{\delta} \quad$ Reynolds number based on viscous penetration depth, $R e_{\delta}=\bar{U}_{\text {peak }} \frac{\sqrt{\frac{v T}{\pi}}}{v}$

$L_{\bar{U}_{e}} \quad$ Stroke ratio based on time-averaged velocity induced by SJA during expulsion stoke, $L_{\bar{U}_{e}}=\frac{\bar{U}_{e}}{D_{o} f}$

$L_{\bar{U}_{\text {ave }}} \quad$ Stroke ratio based on time-averaged velocity induced by SJA during one cycle, $L_{\bar{U}_{\text {ave }}}=\frac{\bar{U}_{\text {ave }}}{D_{o} f}$

$L_{\bar{U}_{\text {ref }}} \quad$ Stroke ratio based on reference velocity, $L_{\bar{U}_{r e f}}=\frac{\bar{U}_{r e f}}{D_{o} f}$

$L_{\bar{U}_{\text {peak }}} \quad$ Stroke ratio based on peak average velocity, $L_{\bar{U}_{\text {ave }}}=\frac{\bar{U}_{\text {peak }}}{D_{o} f}$

$L_{\bar{U}_{\text {mean }}} \quad$ Stroke ratio based on mean velocity, $L_{\bar{U}_{\text {mean }}}=\frac{\bar{U}_{\text {mean }}}{D_{o} f}$

$L_{\bar{U}_{o}} \quad$ Stroke ratio based on slug model, $L_{\bar{U}_{o}}=\frac{0.25 \pi D_{c}^{2} \Delta}{4 l w^{2}}$ 


\begin{tabular}{|c|c|}
\hline$S$ & Stoke Number, $S=\sqrt{\frac{2 \pi f D_{o}^{2}}{v}}$ \\
\hline $\bar{U}_{\text {ref }}$ & Reference velocity \\
\hline $\bar{U}_{p e a k}$ & Peak average velocity induced by SJA \\
\hline $\bar{U}_{\text {mean }}$ & Mean velocity \\
\hline $\bar{V}_{j}$ & Average jet velocity \\
\hline$u_{o}(t)$ & Instantaneous stream-wise space averaged velocity at orifice exit plane \\
\hline$c$ & Speed of sound \\
\hline$v$ & Kinematic viscosity \\
\hline$\mu$ & Dynamic viscosity \\
\hline$S t$ & Strouhal number, $S t=\frac{2 \pi f D_{o}}{U_{o}}$ \\
\hline$u_{p e a k_{-} o p t}$ & Optimum jet peak velocity \\
\hline$\pi_{P_{-} \max }$ & Maximum pressure gradient in the orifice \\
\hline$\rho$ & Density of fluid \\
\hline$K$ & Jet formation constant \\
\hline$\Delta$ & Peak to peak displacement of oscillating diaphragm \\
\hline$x^{*}$ & Streamwise position \\
\hline$V_{c}$ & Volume of cavity \\
\hline
\end{tabular}




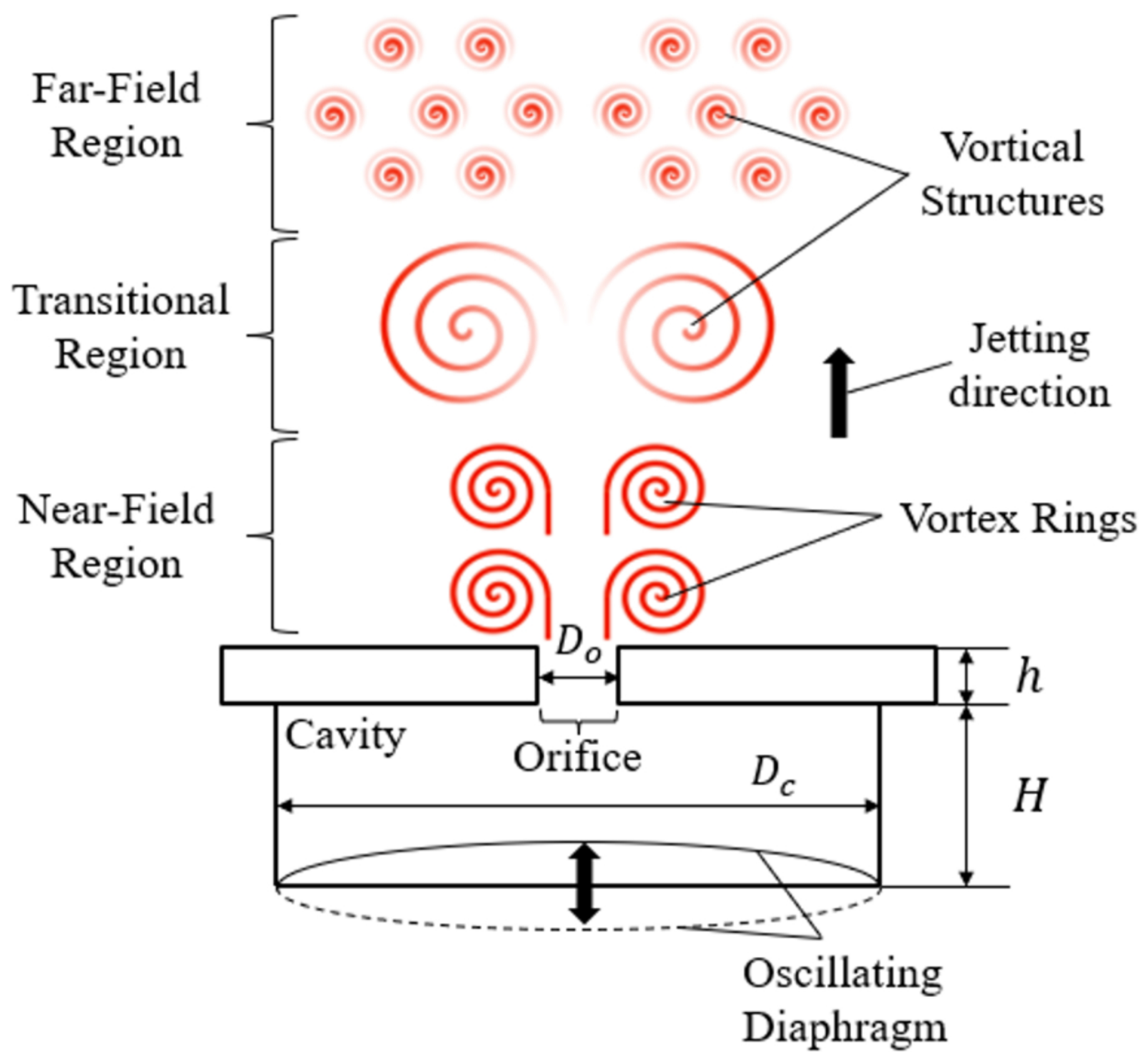


$y$ (short axis)

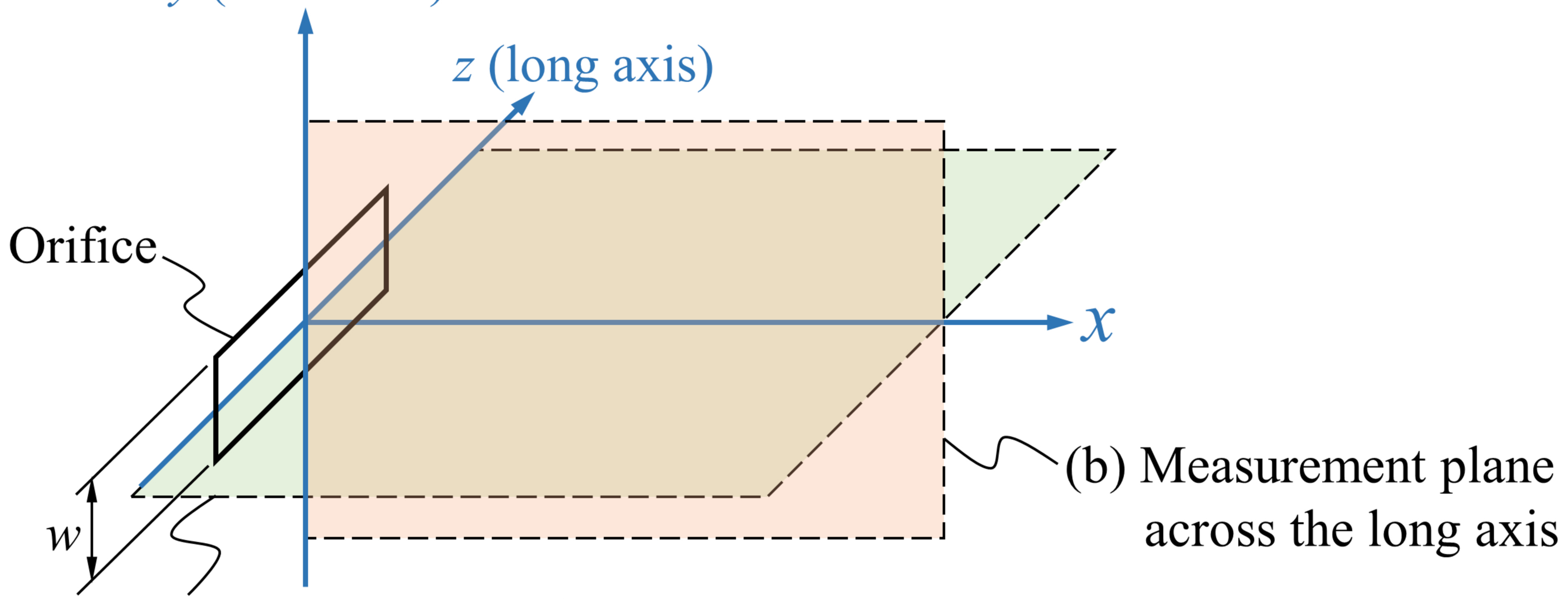

(a) Measurement plane

along the long axis

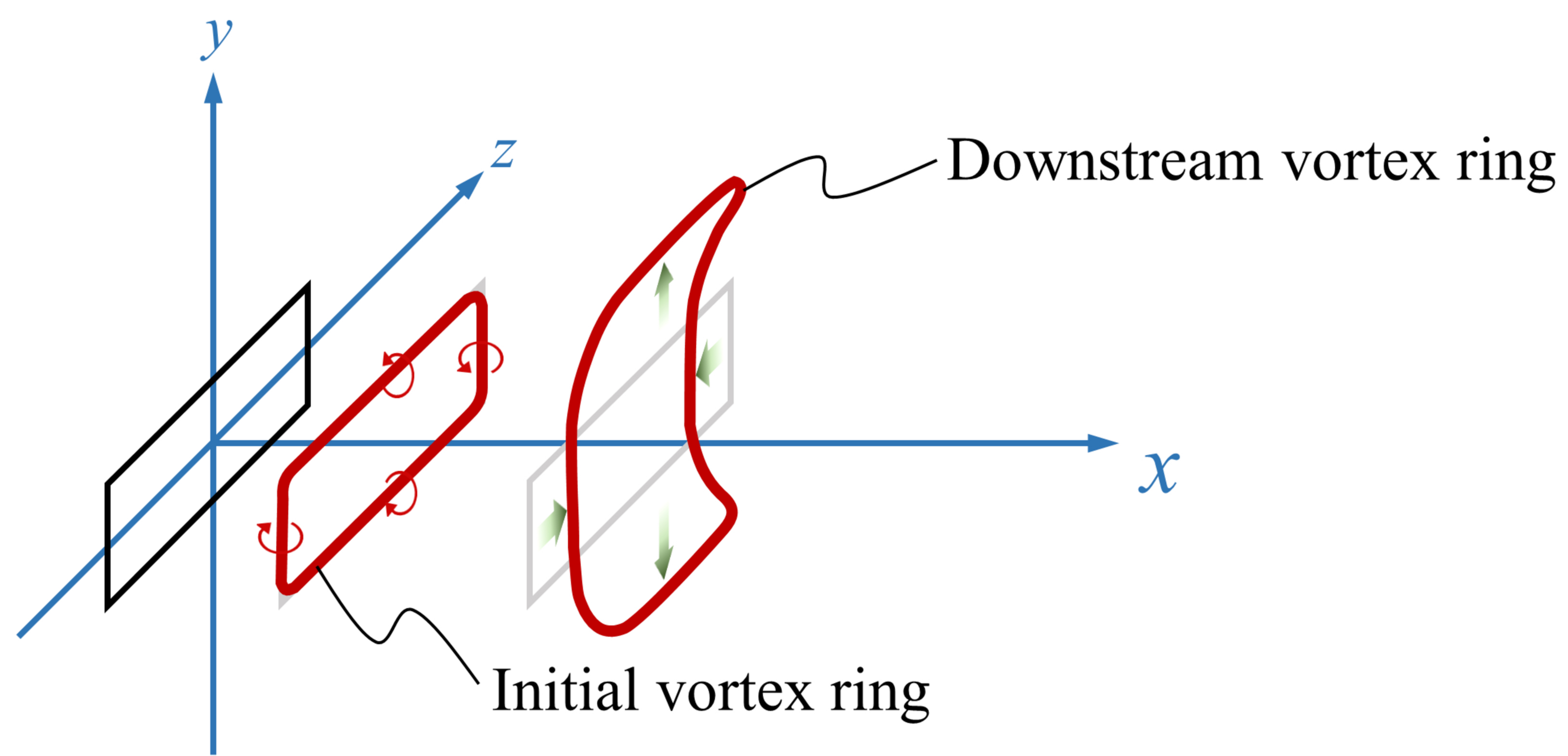


(a)

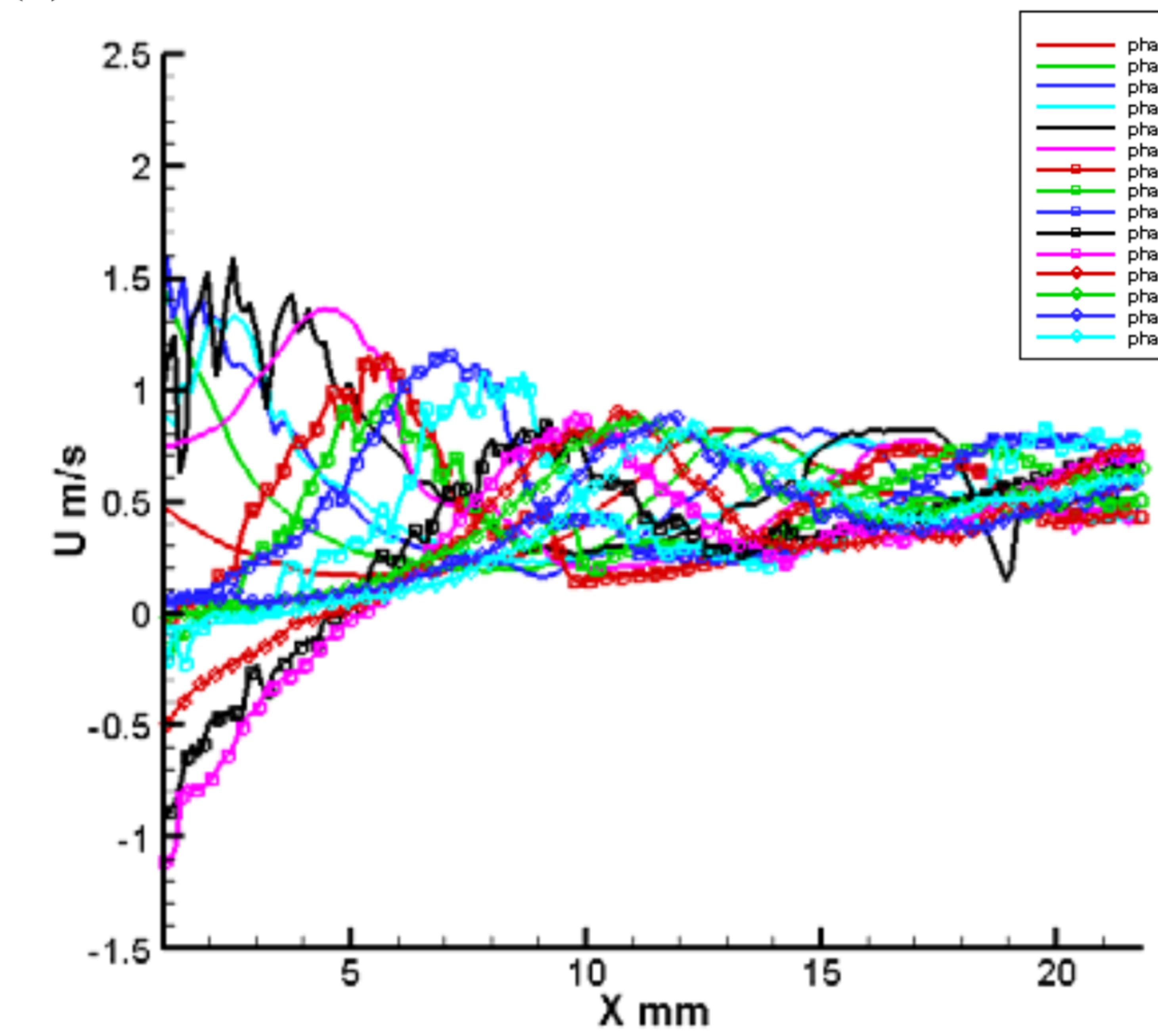

(b)

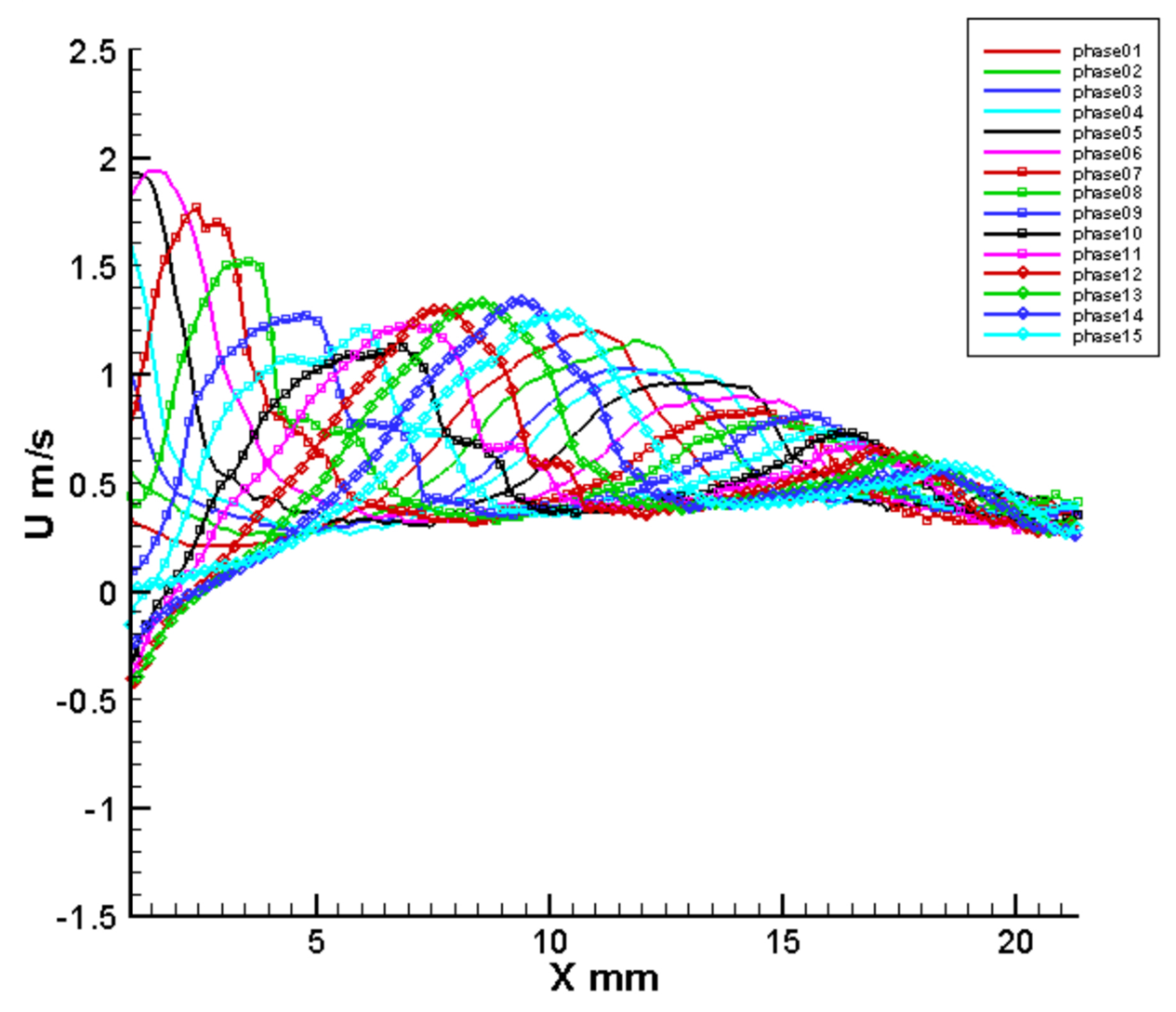

(c)

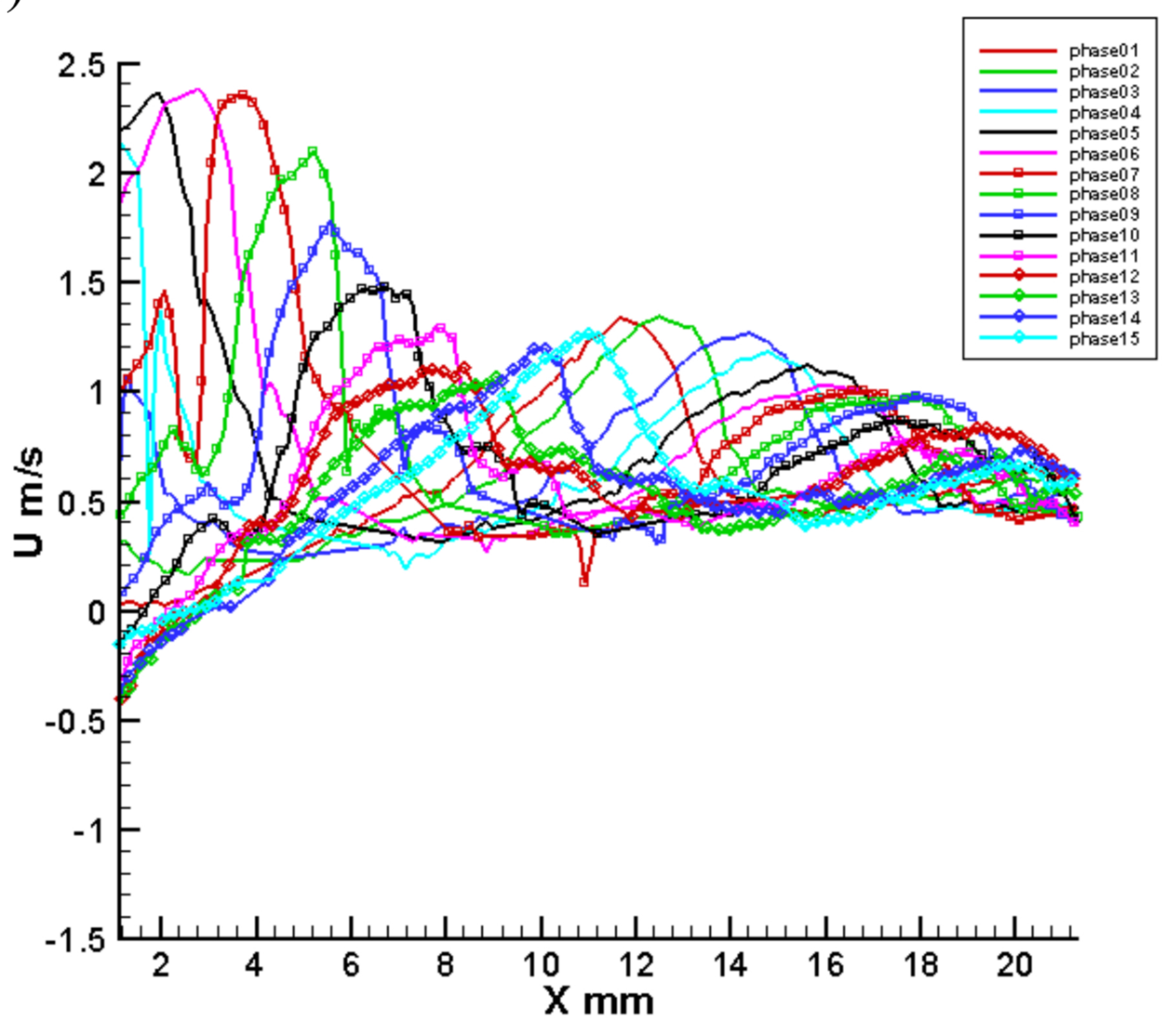

(d)

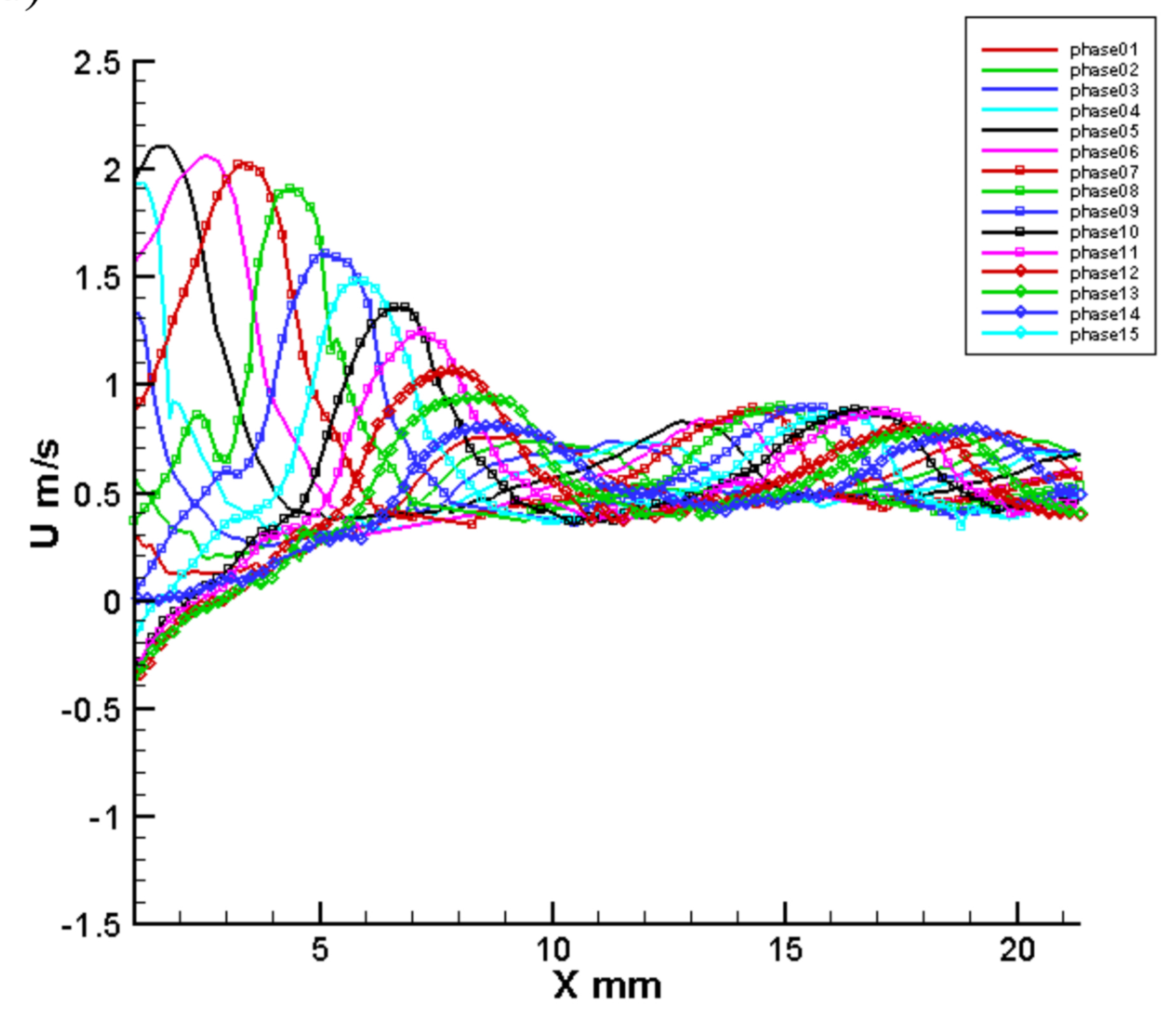



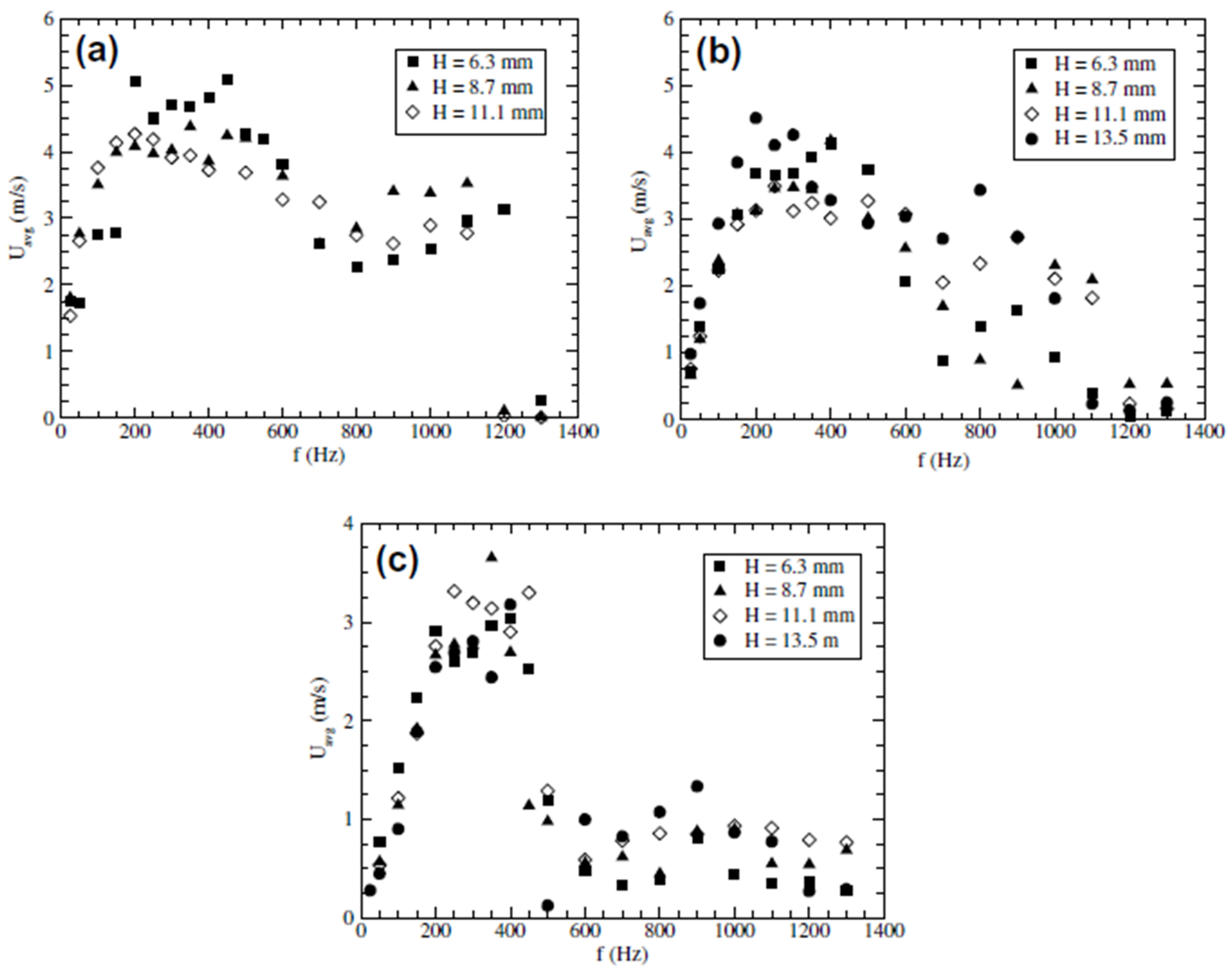
(a)

Orifice duct wall

Flow

from

cavity

Streamline

(b)

Flow

from

cavity

(c)

Flow

from

cavity
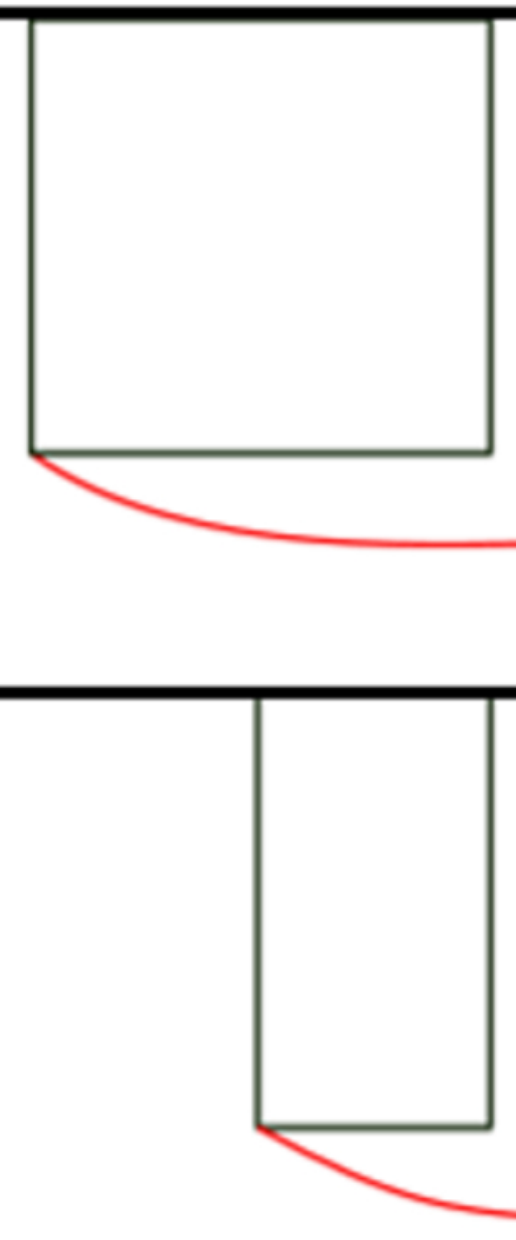


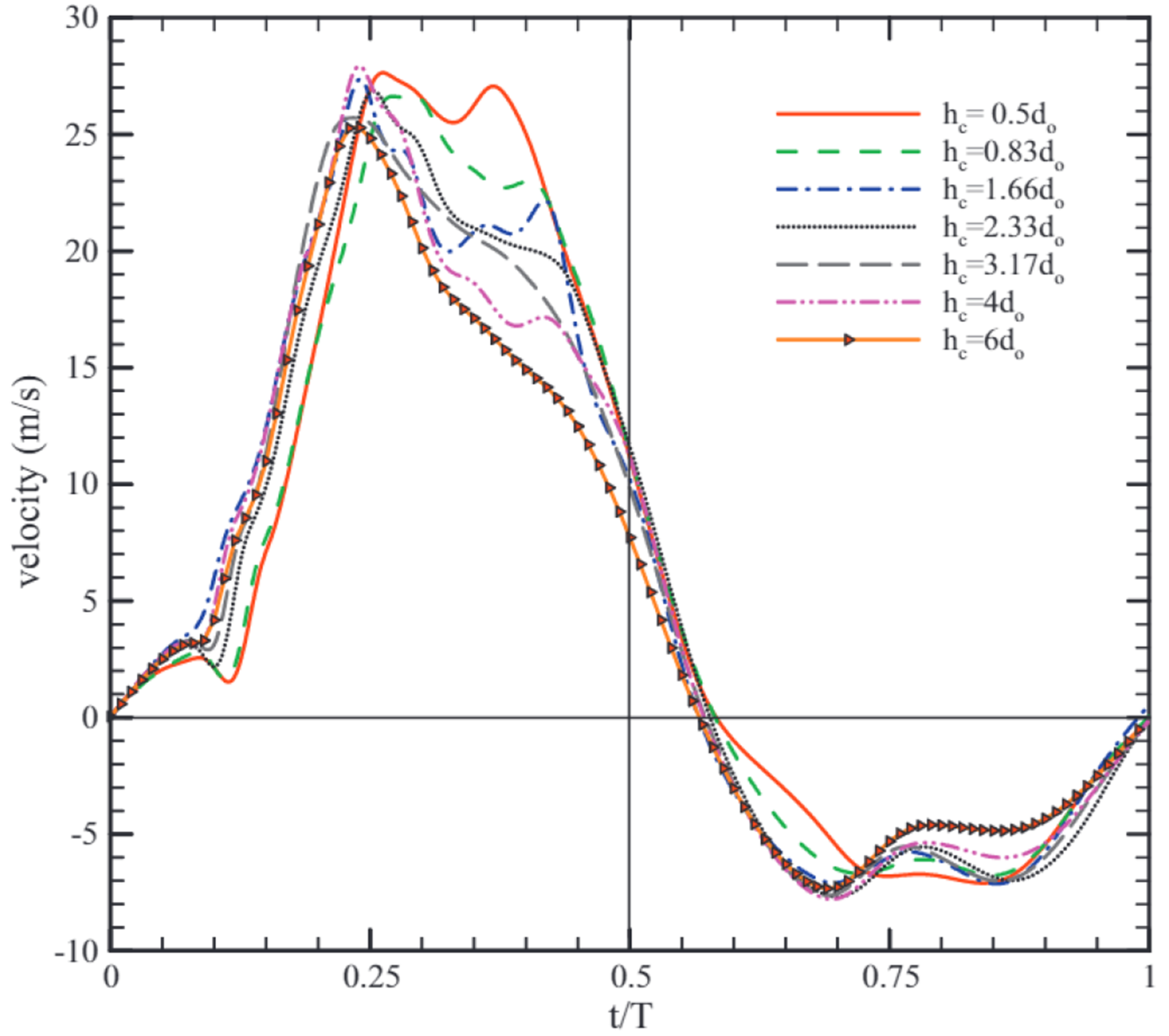



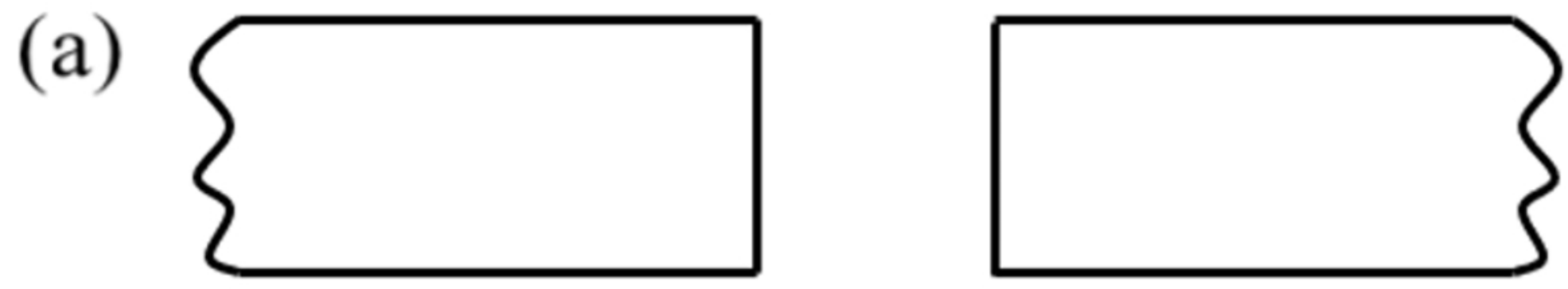

(b)
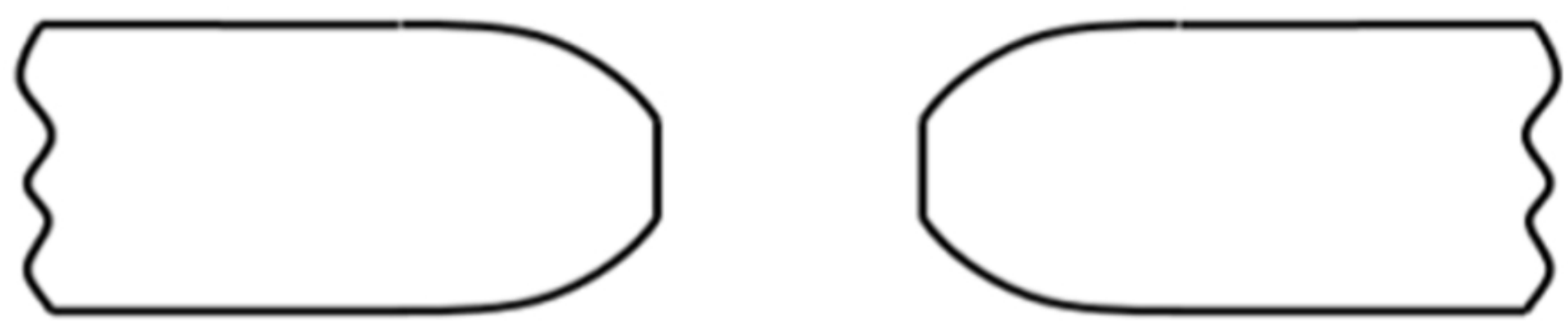

(c)
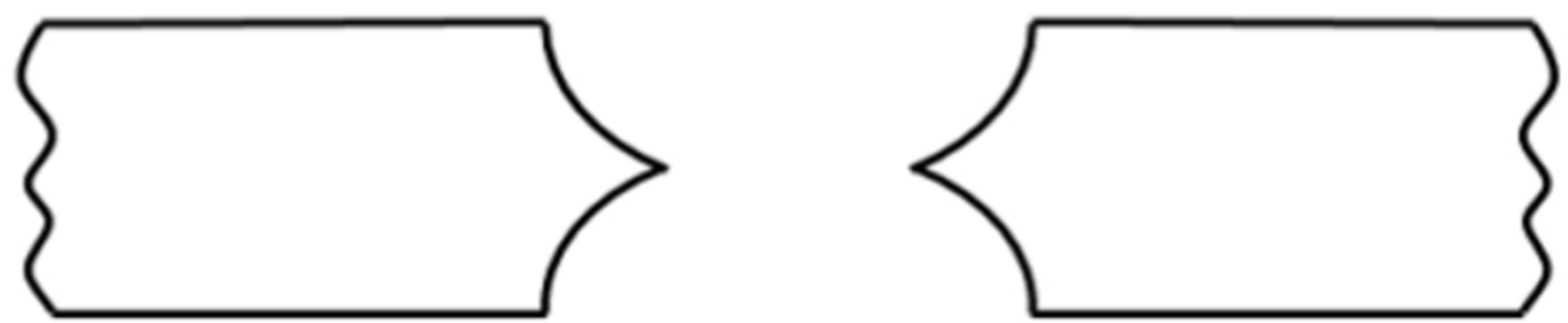
(a)
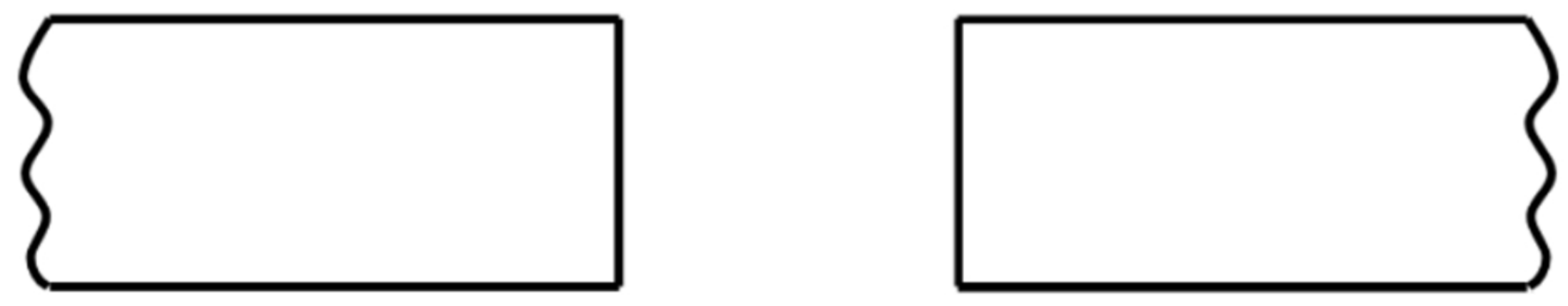

(b)

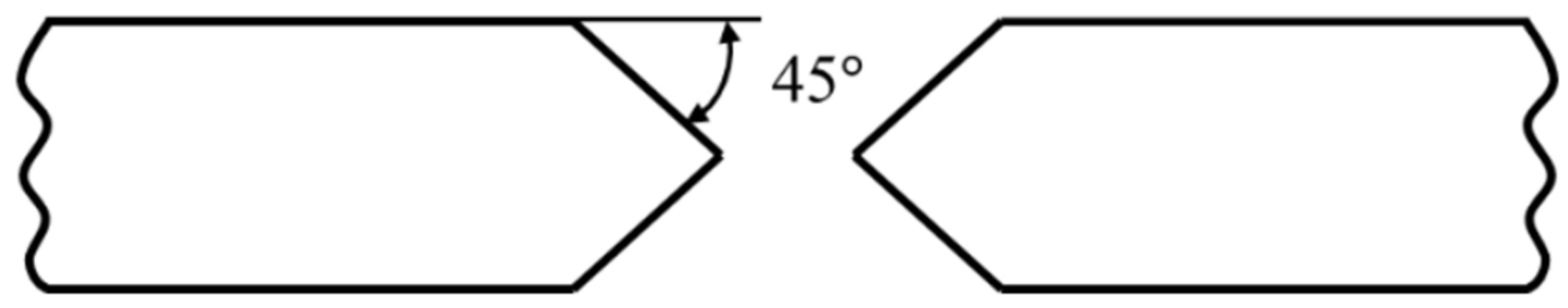



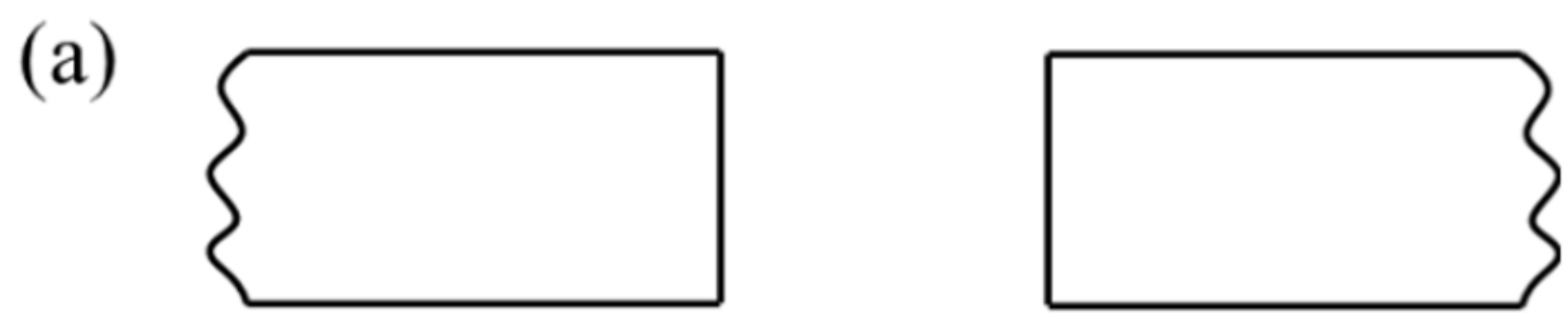

(b)
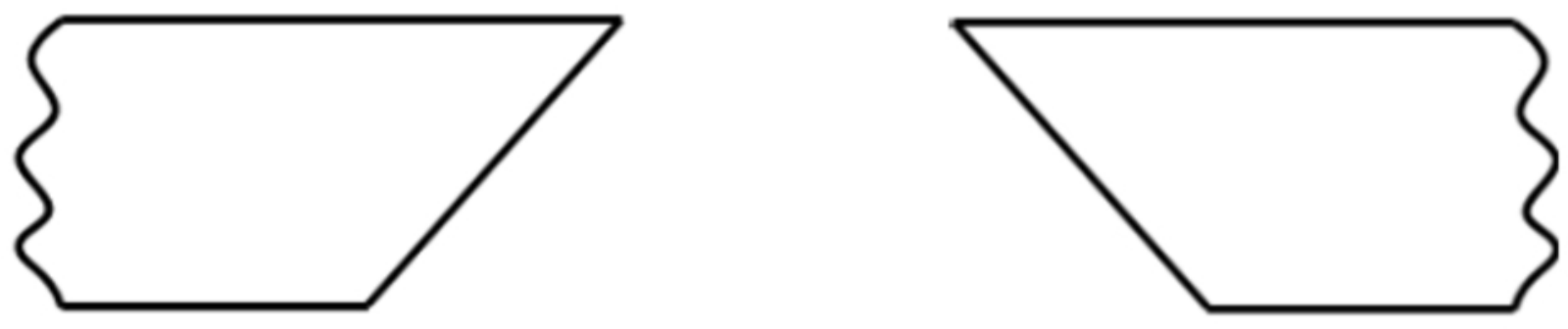

(c)
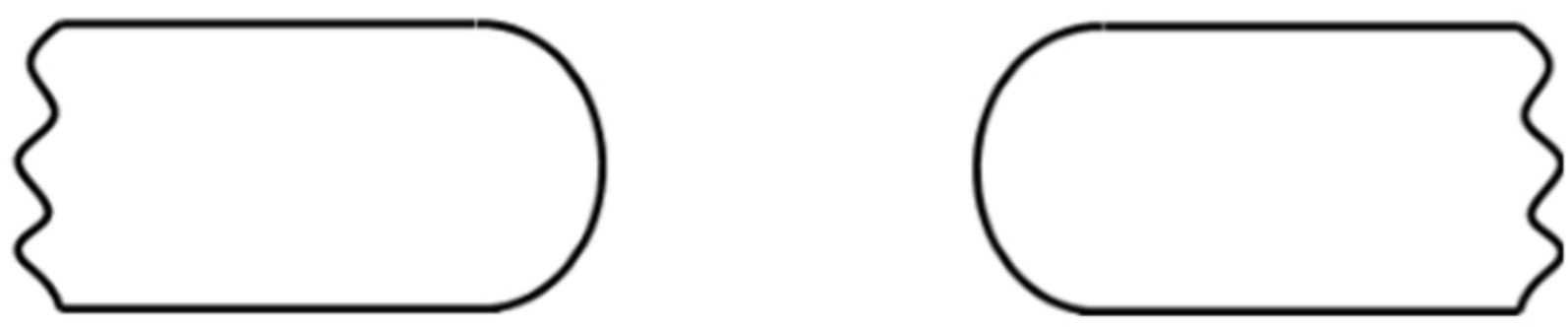
(a)

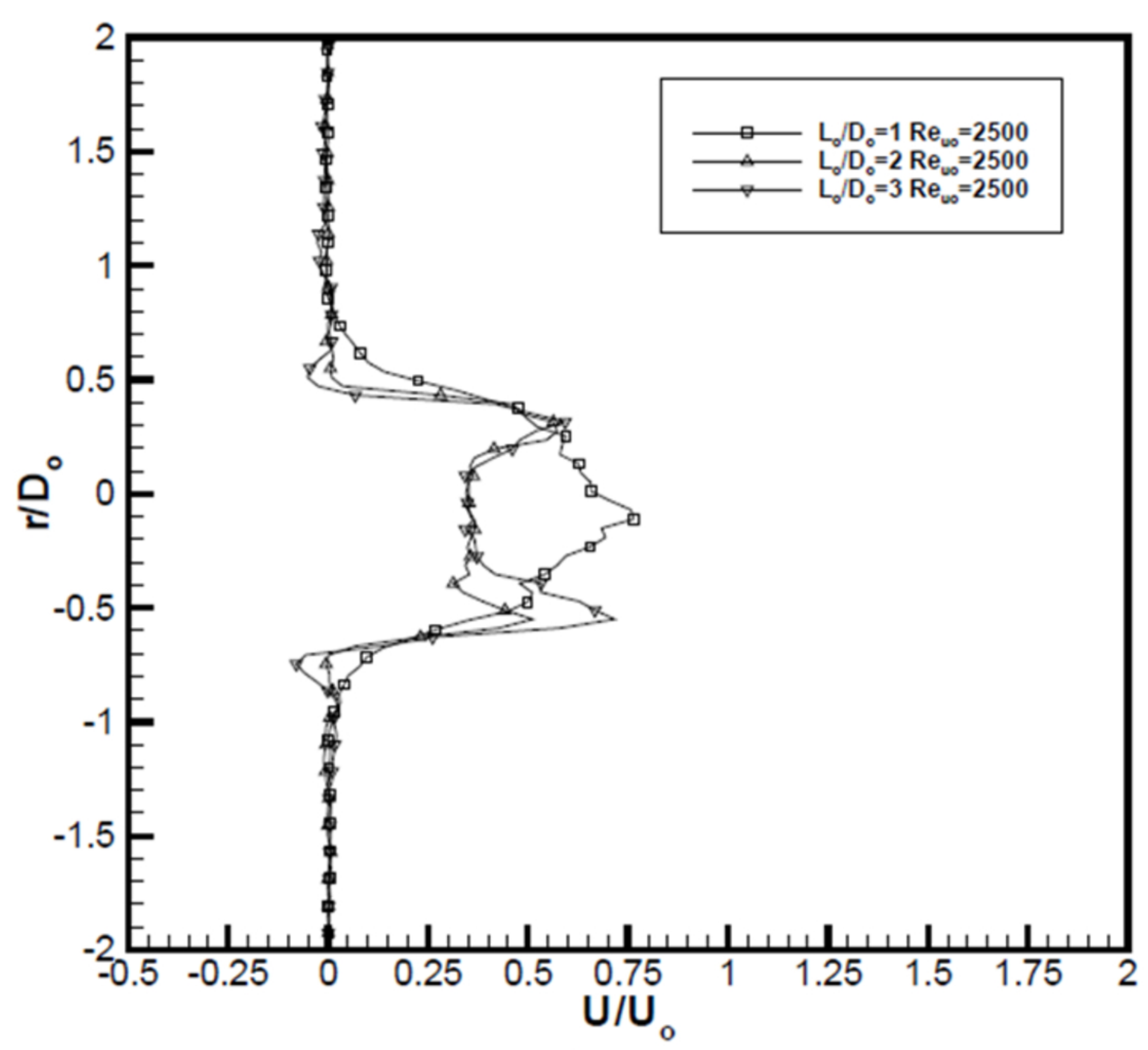

$\phi=36^{\circ}$.

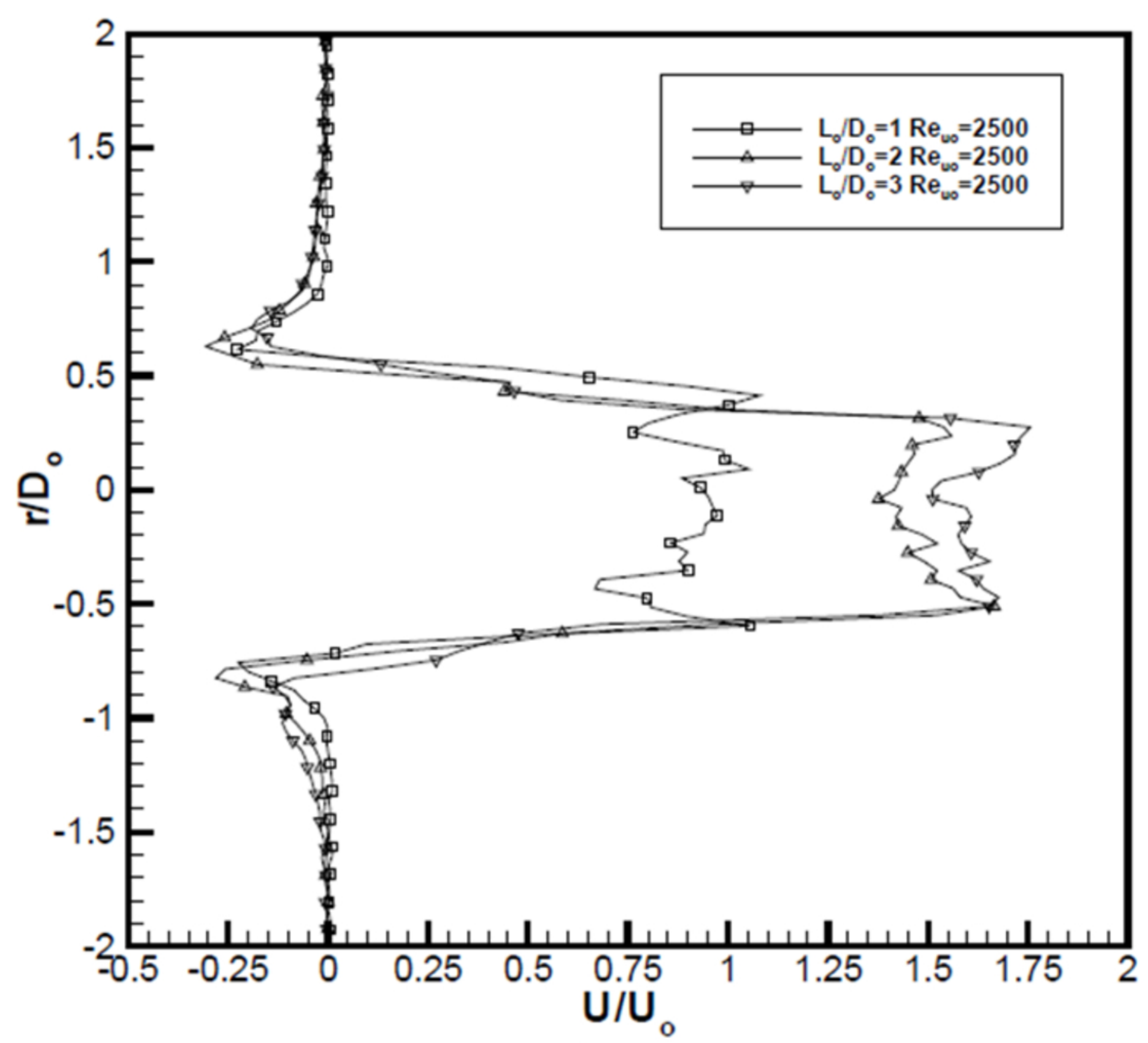

$\phi=90^{\circ}$.

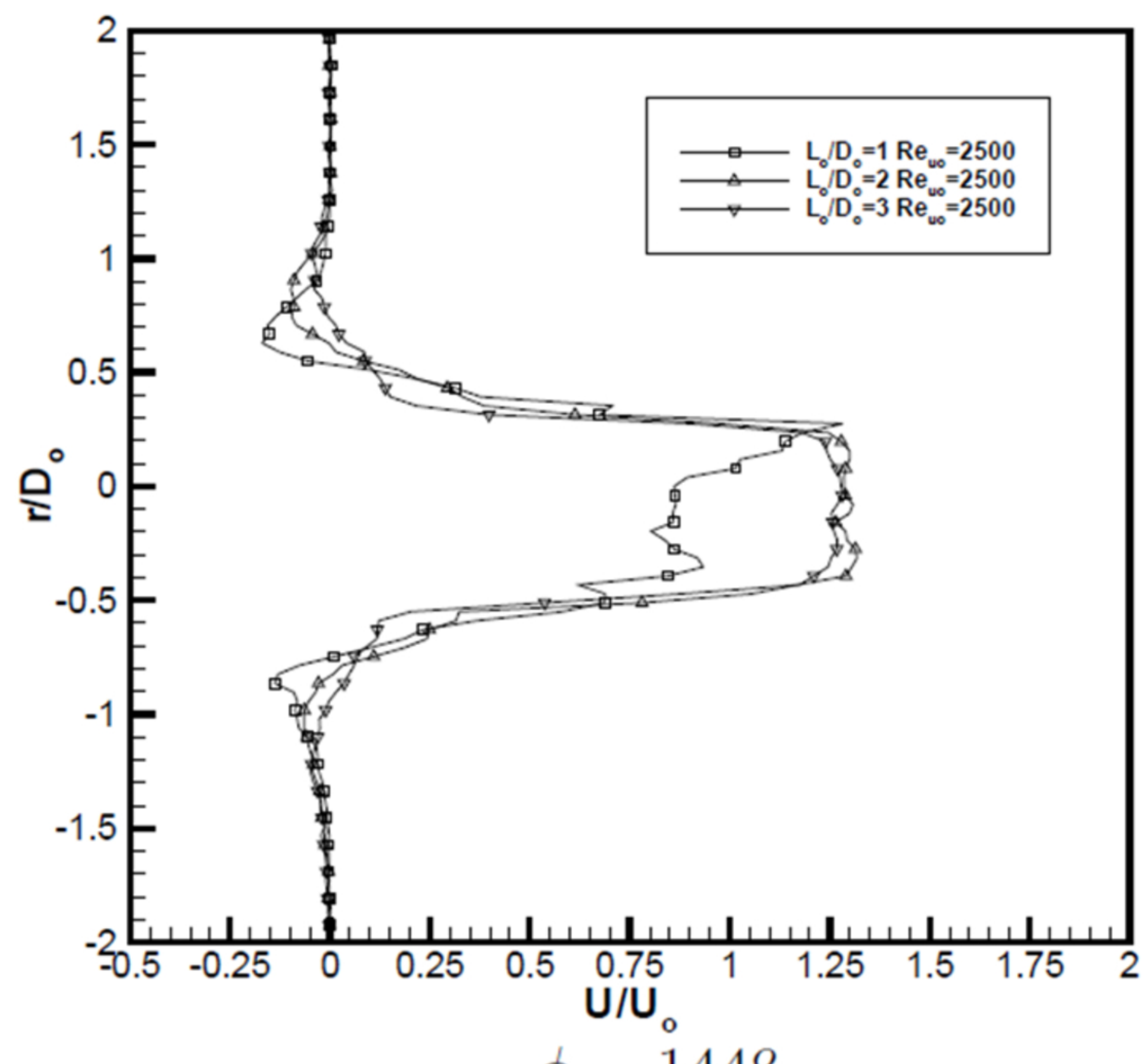

$\phi=144^{\circ}$. (b)

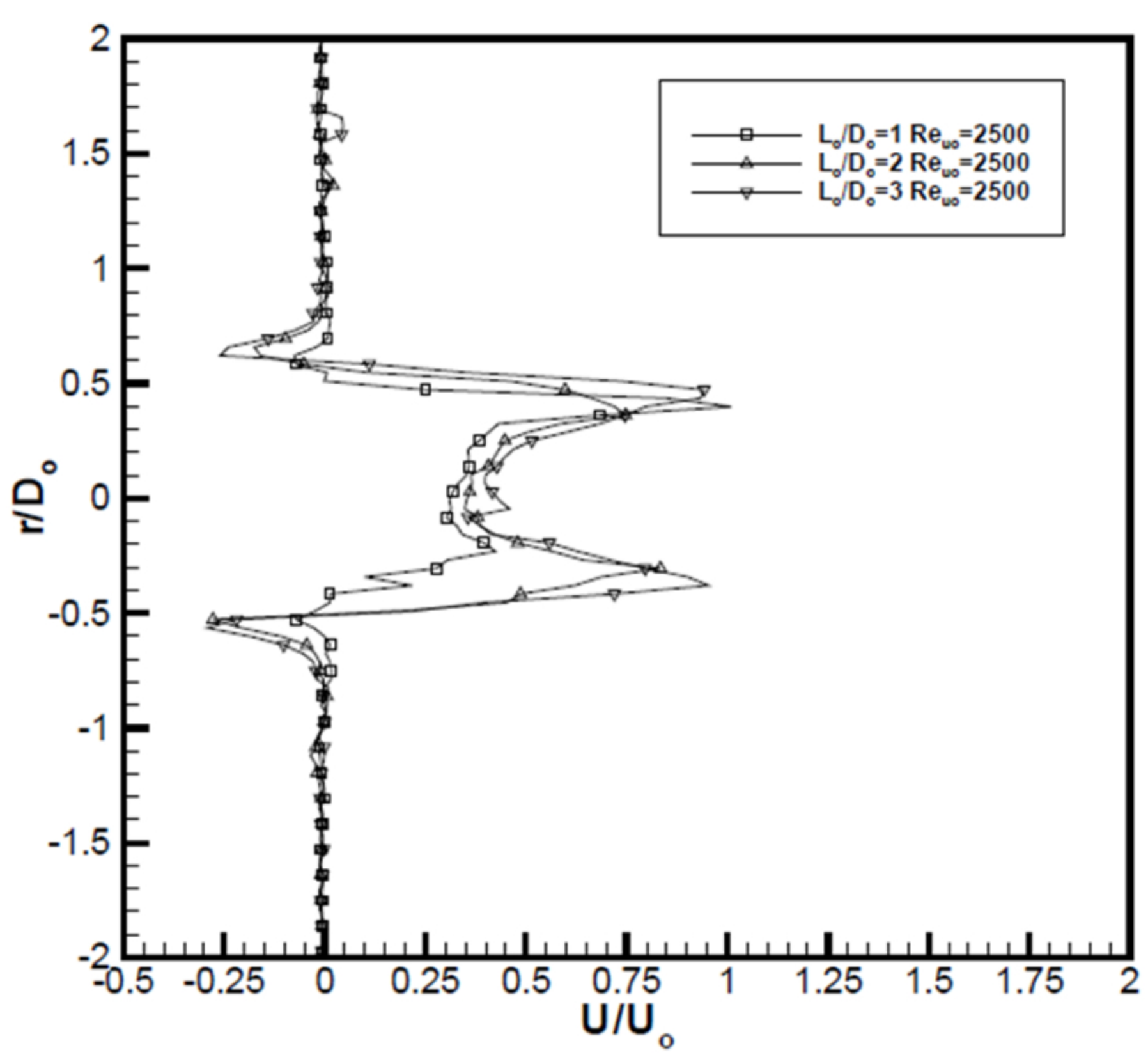

$\phi=36^{\circ}$.

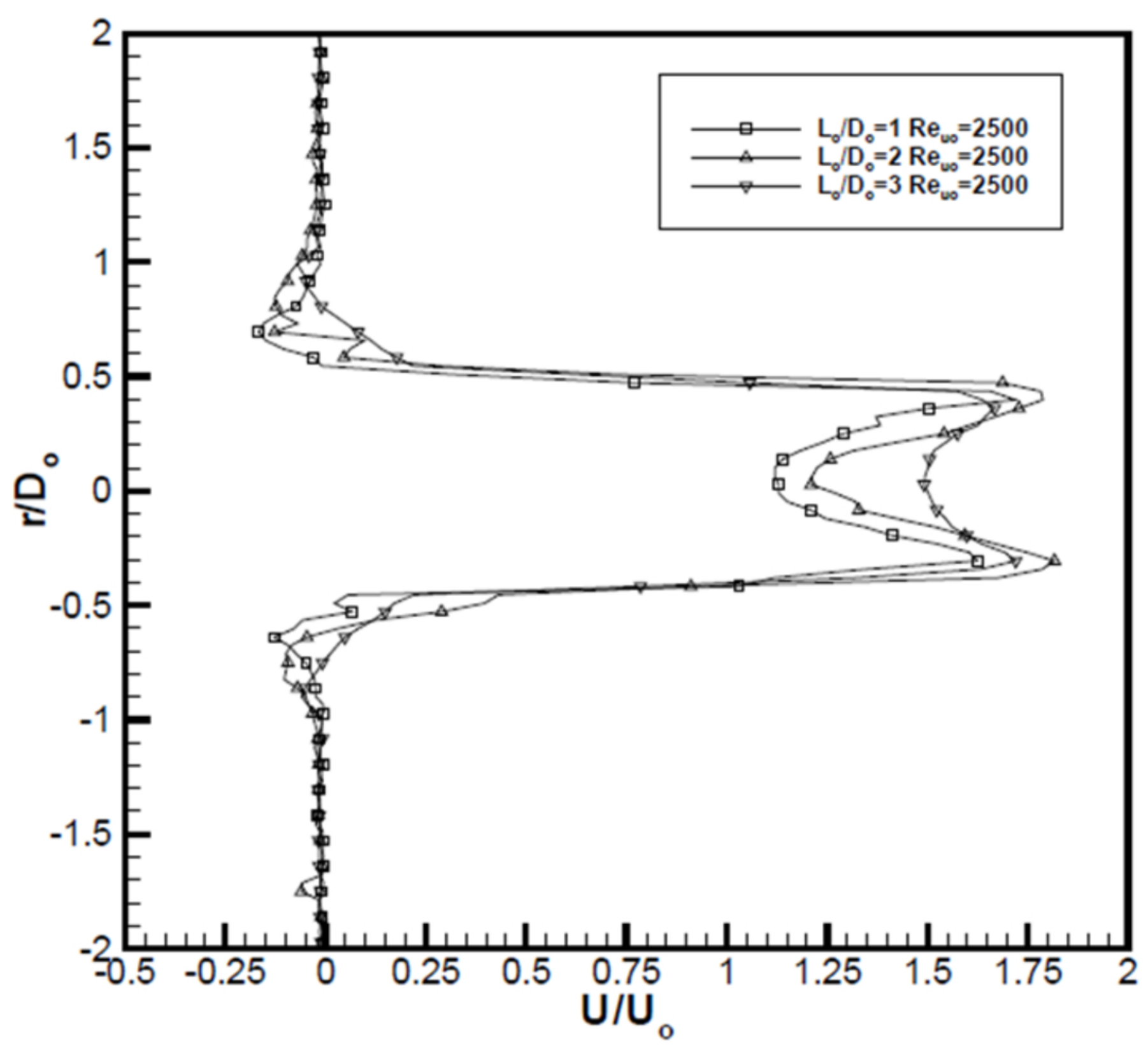

$\phi=90^{\circ}$.

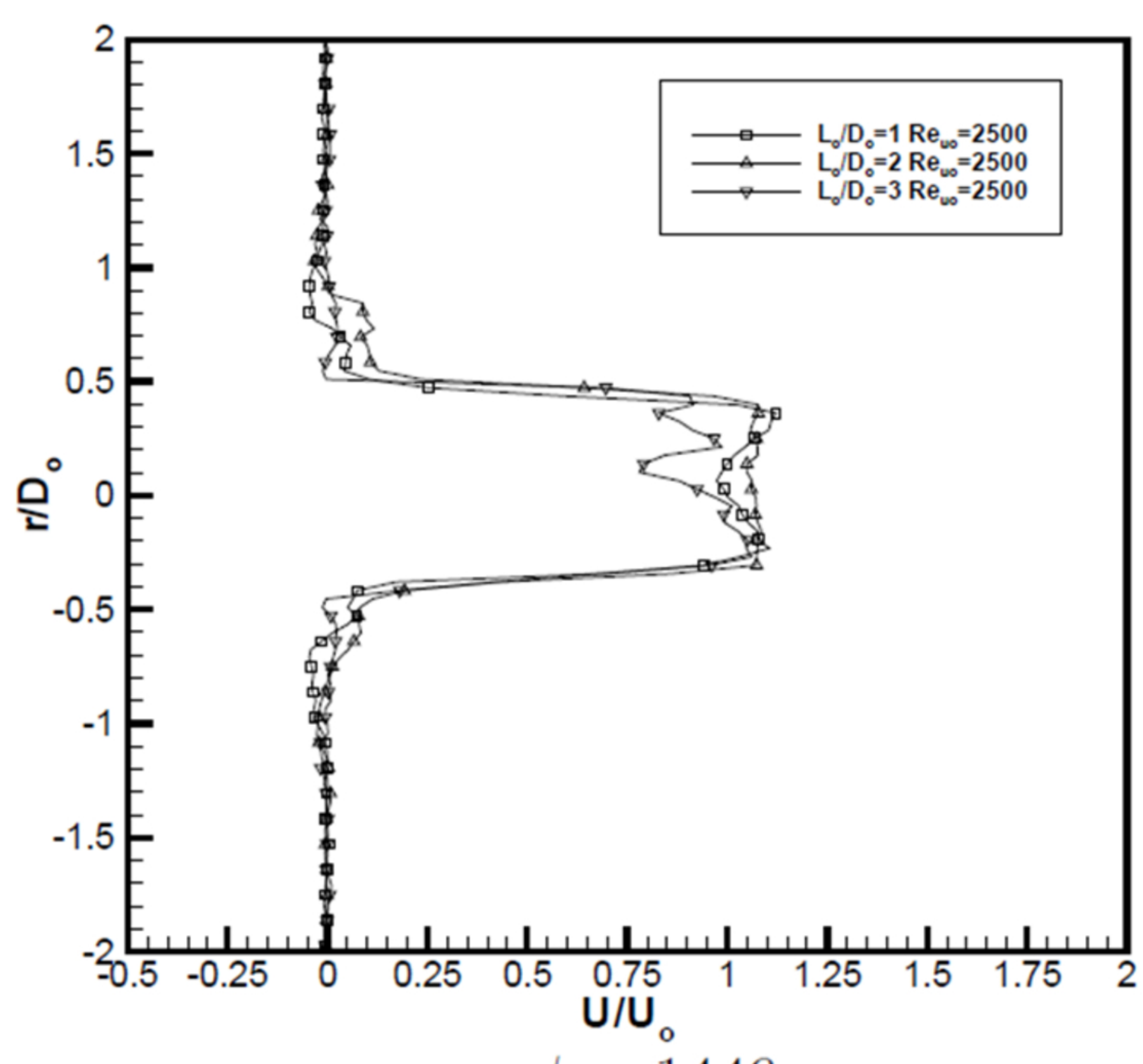

$\phi=144^{\circ}$. 
Nozzle angle

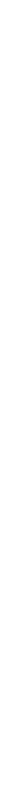

